\title{
Asymptotic analysis in multivariate average case approximation with Gaussian kernels
}

\author{
A. A. Khartov* I. A. Limar ${ }^{\dagger}$
}

March 4, 2021

\begin{abstract}
We consider tensor product random fields $Y_{d}, d \in \mathbb{N}$, whose covariance functions are Gaussian kernels with a given sequence of length scale parameters. The average case approximation complexity $n^{Y_{d}}(\varepsilon)$ is defined as the minimal number of evaluations of arbitrary linear functionals needed to approximate $Y_{d}$, with relative 2-average error not exceeding a given threshold $\varepsilon \in(0,1)$. We investigate the growth of $n^{Y_{d}}(\varepsilon)$ for arbitrary fixed $\varepsilon \in(0,1)$ and $d \rightarrow \infty$. Namely, we find criteria of boundedness for $n^{Y_{d}}(\varepsilon)$ on $d$ and of tending $n^{Y_{d}}(\varepsilon) \rightarrow \infty, d \rightarrow \infty$, for any fixed $\varepsilon \in(0,1)$. In the latter case we obtain necessary and sufficient conditions for the following logarithmic asymptotics

$$
\ln n^{Y_{d}}(\varepsilon)=a_{d}+q(\varepsilon) b_{d}+o\left(b_{d}\right), \quad d \rightarrow \infty,
$$

with any $\varepsilon \in(0,1)$. Here $q:(0,1) \rightarrow \mathbb{R}$ is a non-decreasing function, $\left(a_{d}\right)_{d \in \mathbb{N}}$ is a sequence and $\left(b_{d}\right)_{d \in \mathbb{N}}$ is a positive sequence such that $b_{d} \rightarrow \infty, d \rightarrow \infty$. We show that only special quantiles of self-decomposable distribution functions appear as functions $q$ in a given asymptotics. These general results apply to $n^{Y_{d}}(\varepsilon)$ under particular assumptions on the length scale parameters.
\end{abstract}

Keywords and phrases: average case approximation, multivariate problems, random fields, Gaussian kernels, asymptotic analysis, tractability.

\section{$1 \quad$ Introduction and problem setting}

We consider a multivariate approximation problem in average case setting for special random fields with arbitrary large parametric dimension.

Let $X=\{X(t), t \in \mathbb{R}\}$ be a random process defined on some probability space. Here and below $\mathbb{R}$ denotes the set of real numbers. Suppose that the process has zero mean and the following covariance function

$$
\mathcal{K}_{\sigma}(t, s)=\exp \left\{-\frac{(t-s)^{2}}{2 \sigma^{2}}\right\}, \quad t, s \in \mathbb{R}
$$

\footnotetext{
${ }^{*}$ Smolensk State University, 4 Przhevalsky st., 214000 Smolensk, Russia, e-mail: alexeykhartov@gmail.com

${ }^{\dagger}$ Saint-Petersburg National Research University of Information Technologies, Mechanics and Optics (ITMO University), 49 Kronverksky Pr., 197101 Saint-Petersburg, Russia, e-mail: ivan.limar95@gmail.com
} 
where $\sigma>0$ is a length scale parameter. The process is usually considered as a random element of the space $L_{2}(\mathbb{R}, \mu)$, where $\mu$ is the standard Gaussian measure on $\mathbb{R}$. Covariance operator acts as follows

$$
K_{\sigma} f(t)=\int_{\mathbb{R}} \mathcal{K}_{\sigma}(t, s) f(s) \mu(\mathrm{d} s)=\int_{\mathbb{R}} \mathcal{K}_{\sigma}(t, s) f(s) \frac{e^{-\frac{s^{2}}{2}}}{\sqrt{2 \pi}} \mathrm{d} s, \quad t \in \mathbb{R} .
$$

We consider $d$-variate version of $X$ with arbitrary large $d \in \mathbb{N}$ (set of positive integers). Namely, we consider a zero-mean random field $Y_{d}=\left\{Y_{d}(t), t \in \mathbb{R}^{d}\right\}$ with the following covariance function

$$
\mathcal{K}^{Y_{d}}(t, s)=\prod_{j=1}^{d} \mathcal{K}_{\sigma_{j}}\left(t_{j}, s_{j}\right)=\exp \left\{-\sum_{j=1}^{d} \frac{\left(t_{j}-s_{j}\right)^{2}}{2 \sigma_{j}^{2}}\right\}
$$

where $t=\left(t_{1}, \ldots, t_{d}\right)$ and $s=\left(s_{1}, \ldots, s_{d}\right)$ are from $\mathbb{R}^{d}$. Here $\left(\sigma_{j}\right)_{j \in \mathbb{N}}$ is a given sequence of length scale parameters, which are generally have different values. If every $\mathcal{K}_{\sigma_{j}}$ corresponds to a zero-mean process $X_{j}=\left\{X_{j}(t), t \in \mathbb{R}\right\}$ (defined on some probability space), $j \in \mathbb{N}$, then $Y_{d}$ is called tensor product of $X_{1}, \ldots, X_{d}$ (see [10]). Function (2) is well known as Gaussian kernel, which is often used in numerical computation and statistical learning (see [3], [8], [18], [20], [23]).

For every $d \in \mathbb{N}$ the random field $Y_{d}$ is considered as random element of the space $L_{2}\left(\mathbb{R}^{d}, \mu_{d}\right)$, where $\mu_{d}$ is the standard Gaussian measure on $\mathbb{R}^{d}$. So the space is equipped with the inner product

$$
\langle f, g\rangle_{2, d}=\int_{\mathbb{R}^{d}} f(x) g(x) \mu_{d}(\mathrm{~d} x)=\int_{\mathbb{R}^{d}} f(x) g(x) \frac{1}{(2 \pi)^{d / 2}} \exp \left\{-\frac{1}{2} \sum_{j=1}^{d} x_{j}^{2}\right\} \mathrm{d} x
$$

and the norm

$$
\|f\|_{2, d}=\left(\int_{\mathbb{R}^{d}} f(x)^{2} \mu_{d}(\mathrm{~d} x)\right)^{1 / 2}=\left(\int_{\mathbb{R}^{d}} f(x)^{2} \frac{1}{(2 \pi)^{d / 2}} \exp \left\{-\frac{1}{2} \sum_{j=1}^{d} x_{j}^{2}\right\} \mathrm{d} x\right)^{1 / 2}
$$

where $x=\left(x_{1}, \ldots, x_{d}\right) \in \mathbb{R}^{d}$ in the integrals. The covariance operator $K^{Y_{d}}$ of $Y_{d}$ acts as follows

$$
K^{Y_{d}} f(t)=\int_{\mathbb{R}^{d}} \mathcal{K}^{Y_{d}}(t, s) f(s) \mu_{d}(\mathrm{~d} s)=\int_{\mathbb{R}^{d}} \mathcal{K}^{Y_{d}}(t, s) f(s) \frac{1}{(2 \pi)^{d / 2}} \exp \left\{-\frac{1}{2} \sum_{j=1}^{d} s_{j}^{2}\right\} \mathrm{d} s
$$

where $t=\left(t_{1}, \ldots, t_{d}\right)$ and $s=\left(s_{1}, \ldots, s_{d}\right)$ are from $\mathbb{R}^{d}$.

We consider the average case approximation complexity (approximation complexity for short) of $Y_{d}, d \in \mathbb{N}:$

$$
n^{Y_{d}}(\varepsilon):=\min \left\{n \in \mathbb{N}: e^{Y_{d}}(n) \leqslant \varepsilon e^{Y_{d}}(0)\right\},
$$

where $\varepsilon \in(0,1)$ is a given error threshold, and

$$
e^{Y_{d}}(n):=\inf \left\{\left(\mathbb{E}\left\|Y_{d}-Y_{d}^{(n)}\right\|_{2, d}^{2}\right)^{1 / 2}: Y_{d}^{(n)} \in \mathcal{A}_{n}^{Y_{d}}\right\}
$$


is the smallest 2-average error among all linear approximations of $Y_{d}$ having rank $n \in \mathbb{N}$ ( $\mathbb{E}$ is the expectation). The corresponding classes of linear algorithms are

$$
\mathcal{A}_{n}^{Y_{d}}:=\left\{\sum_{m=1}^{n}\left\langle Y_{d}, \psi_{m}\right\rangle_{2, d} \psi_{m}: \psi_{m} \in L_{2}\left(\mathbb{R}^{d}, \mu_{d}\right)\right\} .
$$

We will deal with the normalized error, i.e. we take into account the quantity:

$$
e^{Y_{d}}(0):=\left(\mathbb{E}\left\|Y_{d}\right\|_{2, d}^{2}\right)^{1 / 2}<\infty,
$$

which is the approximation error of $Y_{d}$ by zero element.

For a given sequence $\left(\sigma_{j}\right)_{j \in \mathbb{N}}$ of length scale parameters in (2) the quantity $n^{Y_{d}}(\varepsilon)$ is considered as a function depending on two variables $d \in \mathbb{N}$ and $\varepsilon \in(0,1)$. There are a lot of results in this direction concerning the tractability (see [15]). They provide necessary and sufficient conditions on $\left(\sigma_{j}\right)_{j \in \mathbb{N}}$ to have upper bounds of given forms for the approximation complexity. The results within the described average case setting can be find in the papers [4], [5], and [12]. The other setting of the worst case was considered in [6], [16], and [21. We will investigate $n^{Y_{d}}(\varepsilon)$ in the different way. Namely, we are interested in the asymptotic behaviour of $n^{Y_{d}}(\varepsilon)$ for arbirarily small fixed $\varepsilon$ and $d \rightarrow \infty$. We are not aware of any asymptotic results in this way specially for random fields with covariance functions (2). There exist a suitable general methods and results from [11], but their application requires an additional analysis. So in fact we will do such analysis in this paper.

We will use the following notation. Let $\mathbb{N}_{0}$ denote the set of non-negative integers. We write $a_{n} \sim b_{n}$ if $a_{n} / b_{n} \rightarrow 1, n \rightarrow \infty$. The indicator $\mathbb{1}(A)$ equals one if $A$ is true and zero if $A$ is false. For any function $f$ we will denote by $\mathcal{C}(f)$ the set of all its continuity points and by $f^{-1}$ the generalized inverse function $f^{-1}(y):=\inf \{x \in \mathbb{R}: f(x) \geqslant y\}$, where $y$ is from the range of $f$. By distribution function $F$ we mean a non-decreasing function $F$ on $\mathbb{R}$ that is right-continuous on $\mathbb{R}, \lim _{x \rightarrow-\infty} F(x)=0$, and $\lim _{x \rightarrow \infty} F(x)=1$.

\section{Preliminaries}

The quantity $n^{Y_{d}}(\varepsilon)$ can be described in terms of the eigenvalues of the covariance operator $K^{Y_{d}}$. Let $\left(\lambda_{m}^{Y_{d}}\right)_{m \in \mathbb{N}}$ denote the sequence of eigenvalues and $\left(\psi_{m}^{Y_{d}}\right)_{m \in \mathbb{N}}$ the corresponding sequence of orthonormal eigenvectors of $K^{Y_{d}}$. The family $\left(\lambda_{m}^{Y_{d}}\right)_{m \in \mathbb{N}}$ is assumed to be ranked in non-increasing order. We have therefore $K^{Y_{d}} \psi_{m}^{Y_{d}}(t)=\lambda_{m}^{Y_{d}} \psi_{m}^{Y_{d}}(t), m \in \mathbb{N}, t \in \mathbb{R}^{d}$. We denote by $\Lambda^{Y_{d}}$ the trace of $K^{Y_{d}}$, i.e. $\Lambda^{Y_{d}}:=\sum_{m=1}^{\infty} \lambda_{m}^{Y_{d}}$.

It is well known (see [2], [19], [22]) that for any $n \in \mathbb{N}$ the following $n$-rank random field

$$
\widetilde{Y}_{d}^{(n)}(t):=\sum_{k=1}^{n}\left\langle Y_{d}, \psi_{k}^{Y_{d}}\right\rangle_{2, d} \psi_{k}^{Y_{d}}(t), \quad t \in \mathbb{R}^{d}
$$

minimizes the 2-average case error. Hence formula (3) is reduced to

$$
n^{Y_{d}}(\varepsilon)=\min \left\{n \in \mathbb{N}: \mathbb{E}\left\|Y_{d}-\widetilde{Y}_{d}^{(n)}\right\|_{2, d}^{2} \leqslant \varepsilon^{2} \mathbb{E}\left\|Y_{d}\right\|_{2, d}^{2}\right\}, \quad d \in \mathbb{N}, \varepsilon \in(0,1) .
$$

Due to (44) and $\mathbb{E}\left\langle Y_{d}, \psi_{m}^{Y_{d}}\right\rangle_{2, d}^{2}=\left\langle\psi_{m}^{Y_{d}}, K^{Y_{d}} \psi_{m}^{Y_{d}}\right\rangle_{2, d}=\lambda_{m}^{Y_{d}}, m \in \mathbb{N}$, we have the needed representation:

$$
n^{Y_{d}}(\varepsilon)=\min \left\{n \in \mathbb{N}: \sum_{m=n+1}^{\infty} \lambda_{m}^{Y_{d}} \leqslant \varepsilon^{2} \Lambda^{Y_{d}}\right\}, \quad d \in \mathbb{N}, \varepsilon \in(0,1) .
$$


We now consider the sequence $\left(\lambda_{m}^{Y_{d}}\right)_{m \in \mathbb{N}}$. It has the following description. Let $\left(\lambda_{\sigma, k}\right)_{k \in \mathbb{N}}$ denote the sequence of eigenvalues (ranked in non-increasing order) of the covariance operator $K_{\sigma}$ defined by (11). This sequence is known (see [16], [18], and [24]):

$$
\lambda_{\sigma, k}=(1-\omega) \omega^{k-1}, \quad k \in \mathbb{N}, \quad \text { where } \quad \omega:=\left(1+\frac{\sigma^{2}}{2}\left(1+\sqrt{1+\frac{4}{\sigma^{2}}}\right)\right)^{-1}
$$

In particular, we have $\lambda_{\sigma, 1}=1-\omega$ and $\sum_{k \in \mathbb{N}} \lambda_{\sigma, k}=1$. It is well known (see [13] and [16]) that, due to the tensor product structure (2) with given $\sigma_{j}, j \in \mathbb{N},\left(\lambda_{m}^{Y_{d}}\right)_{m \in \mathbb{N}}$ is the sequence of numbers

$$
\prod_{j=1}^{d} \lambda_{\sigma_{j}, k_{j}}=\prod_{j=1}^{d}\left(1-\omega_{j}\right) \omega_{j}^{k_{j}-1}, \quad k_{1}, k_{2}, \ldots, k_{d} \in \mathbb{N}
$$

ranked in non-increasing order (see [15]). Here, according to (15), we set

$$
\omega_{j}:=\left(1+\frac{\sigma_{j}^{2}}{2}\left(1+\sqrt{1+\frac{4}{\sigma_{j}^{2}}}\right)\right)^{-1}, \quad j \in \mathbb{N} .
$$

Observe that

$$
\Lambda^{Y_{d}}=\prod_{j=1}^{d} \sum_{k \in \mathbb{N}} \lambda_{\sigma_{j}, k}=1, \quad d \in \mathbb{N}
$$

Thus each of the sequences $\left(\sigma_{j}\right)_{j \in \mathbb{N}}$ and $\left(\omega_{j}\right)_{j \in \mathbb{N}}$ fully determines $\left(\lambda_{m}^{Y_{d}}\right)_{m \in \mathbb{N}}$ and hence $n^{Y_{d}}(\varepsilon)$ for any $d \in \mathbb{N}$ and $\varepsilon \in(0,1)$.

\section{General results}

Before proceeding to the asymptotic analysis of the quantity $n^{Y_{d}}(\varepsilon)$, we find criteria of its boundedness and unboundedness on $d$ for any fixed $\varepsilon \in(0,1)$. The following propositions show that for any fixed $\varepsilon \in(0,1)$ either the quantity $n^{Y_{d}}(\varepsilon)$ is a bounded function on $d \in \mathbb{N}$ or it tends to infinity as $d \rightarrow \infty$.

Proposition 1 The following conditions are equivalent:

(i) $\sup _{d \in \mathbb{N}} n^{Y_{d}}(\varepsilon)<\infty$ for every $\varepsilon \in(0,1)$;

(ii) $\sum_{j=1}^{\infty} \omega_{j}<\infty$;

(iii) $\sum_{j=1}^{\infty} \sigma_{j}^{-2}<\infty$.

Proof of Proposition 1. By Proposition 5 from [11], the relation $\sup _{d \in \mathbb{N}} n^{Y_{d}}(\varepsilon)<\infty, \varepsilon \in(0,1)$, is equivalent to convergence of the following series

$$
\sum_{j=1}^{\infty} \sum_{k=2}^{\infty} \frac{\lambda_{\sigma_{j}, k}}{\lambda_{\sigma_{j}, 1}}=\sum_{j=1}^{\infty} \sum_{k=2}^{\infty} \omega_{j}^{k-1}=\sum_{j=1}^{\infty} \frac{\omega_{j}}{1-\omega_{j}}=\sum_{j=1}^{\infty} \frac{2}{\sigma_{j}^{2}+\sqrt{\sigma_{j}^{4}+4 \sigma_{j}^{2}}}
$$


Since $\omega_{j} \in(0,1)$, the convergence of $\sum_{j=1}^{\infty} \frac{\omega_{j}}{1-\omega_{j}}$ implies the convergence of $\sum_{j=1}^{\infty} \omega_{j}$. Next, if $\sum_{j=1}^{\infty} \omega_{j}<$ $\infty$, then $\omega_{j} \rightarrow 0$ and hence $\frac{\omega_{j}}{1-\omega_{j}} \sim \omega_{j}, j \rightarrow \infty$. So we have $\sum_{j=1}^{\infty} \frac{\omega_{j}}{1-\omega_{j}}<\infty$.

It is easily seen that the convergence of $\sum_{j=1}^{\infty} \sigma_{j}^{-2}$ implies the convergence of (7). Next, if (77) converges, then $\sigma_{j} \rightarrow \infty$ and $\sigma_{j}^{2}+\sqrt{\sigma_{j}^{4}+4 \sigma_{j}^{2}} \sim 2 \sigma_{j}^{2}, j \rightarrow \infty$. Hence we get the convergence of $\sum_{j=1}^{\infty} \sigma_{j}^{-2}$.

Proposition 2 The following conditions are equivalent:

(i) $\lim _{d \rightarrow \infty} n^{Y_{d}}(\varepsilon)=\infty$ for every $\varepsilon \in(0,1)$;

(ii) $\sum_{j=1}^{\infty} \omega_{j}=\infty$;

(iii) $\sum_{j=1}^{\infty} \sigma_{j}^{-2}=\infty$.

Proof of Proposition 2. According to Proposition 4 and 5 from [1], for any $\varepsilon \in(0,1)$ either $\sup _{d \in \mathbb{N}} n^{Y_{d}}(\varepsilon)<\infty$ or $\lim _{d \rightarrow \infty} n^{Y_{d}}(\varepsilon)=\infty$. Hence the latter is equivalent to divergence of each of the series $\sum_{j=1}^{\infty} \omega_{j}$ and $\sum_{j=1}^{\infty} \sigma_{j}^{-2}$ by Proposition 1 .

It is known (see [11]) that for wide class of tensor product random fields the quantity $n^{Y_{d}}(\varepsilon)$ has the logarithmic asymptotics of the form (8) below. Our next theorem shows that the function $q$ can be only a special quantile of self-decomposable distribution function in such asymptotics (see [7], [17], or [11], Appendix). Recall that self-decomposable distribution functions are completely described by the triplet $(c, v, L)$ from spectral representation of their characteristic functions, where $c \in \mathbb{R}$ is a shift parameter, $v>0$ is the Gaussian component, $L$ is the Lévy spectral function (see [11], Appendix).

Theorem 1 Let $\left(a_{d}\right)_{d \in \mathbb{N}}$ be a sequence, $\left(b_{d}\right)_{d \in \mathbb{N}}$ be a positive sequence such that $b_{d} \rightarrow \infty, d \rightarrow \infty$. Let a non-increasing function $q:(0,1) \rightarrow \mathbb{R}$ and a distribution function $G$ satisfy the equation $q(\varepsilon)=$ $G^{-1}\left(1-\varepsilon^{2}\right)$ for all $\varepsilon \in \mathcal{C}(q)$. Suppose that the following asymptotics holds

$$
\forall \varepsilon \in \mathcal{C}(q) \quad \ln n^{Y_{d}}(\varepsilon)=a_{d}+q(\varepsilon) b_{d}+o\left(b_{d}\right), \quad d \rightarrow \infty
$$

Then $G$ is self-decomposable with zero Lévy spectral function on $(-\infty, 0)$.

Proof of Theorem 1. Due to Theorem 1 from [11], the condition (8) is equivalent to the convergence

$$
\lim _{d \rightarrow \infty} G_{d}(x)=G(x), \quad x \in \mathcal{C}(G)
$$

where

$$
G_{d}(x):=\sum_{m \in \mathbb{N}} \lambda_{m}^{Y_{d}} \mathbb{1}\left(\lambda_{m}^{Y_{d}} \geqslant e^{-a_{d}-b_{d} x}\right), \quad x \in \mathbb{R}, \quad d \in \mathbb{N} .
$$

It was shown in [11] that $G_{d}, d \in \mathbb{N}$, can be considered as the following distribution functions

$$
G_{d}(x)=\mathbb{P}\left(\frac{\sum_{j=1}^{d} U_{j}-a_{d}}{b_{d}} \leqslant x\right), \quad x \in \mathbb{R}, \quad d \in \mathbb{N},
$$


where $U_{j}, j \in \mathbb{N}$, are independent random variables on some probability space with the measure $\mathbb{P}$. Here $U_{j}, j \in \mathbb{N}$, have the following distribution

$$
\mathbb{P}\left(U_{j}=\left|\ln \lambda_{\sigma_{j}, k}\right|\right)=\lambda_{\sigma_{j}, k}, \quad k \in \mathbb{N}, \quad j \in \mathbb{N}
$$

Now we center these variables in the following way: $\hat{U}_{j}:=U_{j}-\left|\ln \lambda_{\sigma_{j}, 1}\right|, j \in \mathbb{N}$. So we have

$$
\mathbb{P}\left(\hat{U}_{j}=\left|\ln \lambda_{\sigma_{j}, k}\right|-\left|\ln \lambda_{\sigma_{j}, 1}\right|\right)=\lambda_{\sigma_{j}, k}, \quad k \in \mathbb{N}, \quad j \in \mathbb{N},
$$

i.e.

$$
\mathrm{P}\left(\hat{U}_{j}=k\left|\ln \omega_{j}\right|\right)=\left(1-\omega_{j}\right) \omega_{j}^{k}, \quad k \in \mathbb{N}_{0}, \quad j \in \mathbb{N} .
$$

Here $\hat{U}_{j} \geqslant 0, j \in \mathbb{N}$. Next, we set

$$
\hat{a}_{d}:=a_{d}-\sum_{j=1}^{d}\left|\ln \lambda_{\sigma_{j}, 1}\right|=a_{d}-\sum_{j=1}^{d}\left|\ln \left(1-\omega_{j}\right)\right|, \quad d \in \mathbb{N} .
$$

Then

$$
G_{d}(x)=\mathbb{P}\left(\frac{\sum_{j=1}^{d} \hat{U}_{j}-\hat{a}_{d}}{b_{d}} \leqslant x\right), \quad x \in \mathbb{R}, \quad d \in \mathbb{N}
$$

For any $d \in \mathbb{N}, j \in\{1, \ldots, d\}$ and $x>0$ we consider the following distribution tails:

$$
\mathbb{P}\left(\left|\hat{U}_{j}\right|>x b_{d}\right)=\mathbb{P}\left(\hat{U}_{j}>x b_{d}\right)=\sum_{\substack{k \in \mathbb{N}_{0}: \\ k\left|\ln \omega_{j}\right|>x b_{d}}}\left(1-\omega_{j}\right) \omega_{j}^{k}=\omega_{j}^{k_{j, d}(x)},
$$

where $k_{j, d}(x):=\min \left\{k \in \mathbb{N}_{0}: k\left|\ln \omega_{j}\right|>x b_{d}\right\}$. Since $k_{j, d}(x)\left|\ln \omega_{j}\right|>x b_{d}$, we have

$$
\mathbb{P}\left(\left|\hat{U}_{j}\right|>x b_{d}\right)<e^{-x b_{d}}, \quad d \in \mathbb{N}, \quad j \in\{1, \ldots, d\}, \quad x>0 .
$$

Due to $b_{d} \rightarrow \infty, d \rightarrow \infty$, we obtain

$$
\max _{j \in\{1, \ldots, d\}} \mathbb{P}\left(\left|\hat{U}_{j}\right|>x b_{d}\right) \rightarrow 0, \quad d \rightarrow \infty
$$

This is the condition of uniform negligibility of $\hat{U}_{j} / b_{d}$ in the sums $\left(\sum_{j=1}^{n} \hat{U}_{j}-\hat{a}_{d}\right) / b_{d}$. It is known, that under this condition and (11) the limit distribution in (9) is self-decomposable (see [17], p. 101, or [11], Theorem 10). Let $L$ denote the Lévy spectral function of $G$. Due to the non-negativity of $\hat{U}_{j}$, we have $L(x)=0, x<0$ (see [7], p. 124, or [11, Theorem 11).

The next theorem provides a criterion for the asymptotics (8), where $q$ is a quantile of a given self-decomposable distribution function. This is the main result of the paper.

Theorem 2 Let $\left(a_{d}\right)_{d \in \mathbb{N}}$ be a sequence, $\left(b_{d}\right)_{d \in \mathbb{N}}$ be a positive sequence such that $b_{d} \rightarrow+\infty, d \rightarrow \infty$. Let $G$ be a self-decomposable distribution function with triplet $(c, v, L)$ such that $L(x)=0, x<0$. 
Let a non-increasing function $q:(0,1) \rightarrow \mathbb{R}$ satysfy the equation $q(\varepsilon)=G^{-1}\left(1-\varepsilon^{2}\right)$ for all $\varepsilon \in(0,1)$. For the asymptotics

$$
\forall \varepsilon \in(0,1) \quad \ln n^{Y_{d}}(\varepsilon)=a_{d}+q(\varepsilon) b_{d}+o\left(b_{d}\right), \quad d \rightarrow \infty
$$

the following ensemble of conditions is necessary and sufficient:

$$
\begin{aligned}
& \text { (A) } \quad \lim _{d \rightarrow \infty} \sum_{\substack{j=1, \ldots, d \\
\left|\ln \omega_{j}\right|>\tau b_{d}}} \omega_{j}=-L(\tau), \quad \tau>0 ; \\
& \text { (B) } \quad \lim _{d \rightarrow \infty} \frac{1}{b_{d}}\left(\sum_{\substack{j=1, \ldots, d \\
\left|\ln \omega_{j}\right| \leqslant \tau b_{d}}} \frac{\left|\ln \omega_{j}\right| \omega_{j}}{1-\omega_{j}}-\hat{a}_{d}\right)=c+\gamma_{\tau}, \quad \tau>0 ; \\
& \text { (C) } \quad \lim _{\tau \rightarrow 0} \frac{\lim }{d \rightarrow \infty} \frac{1}{b_{d}^{2}} \sum_{\substack{j=1, \ldots, d \\
\left|\ln \omega_{j}\right| \leqslant \tau b_{d}}} \frac{\left|\ln \omega_{j}\right|^{2} \omega_{j}}{\left(1-\omega_{j}\right)^{2}}=\lim _{\tau \rightarrow 0} \varlimsup_{d \rightarrow \infty} \frac{1}{b_{d}^{2}} \sum_{\substack{j=1, \ldots, d \\
\left|\ln \omega_{j}\right| \leqslant \tau b_{d}}} \frac{\left|\ln \omega_{j}\right|^{2} \omega_{j}}{\left(1-\omega_{j}\right)^{2}}=v ;
\end{aligned}
$$

where

$$
\begin{aligned}
& \gamma_{\tau}:=\int_{0}^{\tau} \frac{y^{3} \mathrm{~d} L(y)}{1+y^{2}}-\int_{\tau}^{+\infty} \frac{y \mathrm{~d} L(y)}{1+y^{2}}, \quad \tau>0, \\
& \hat{a}_{d}:=a_{d}-\sum_{j=1}^{d}\left|\ln \left(1-\omega_{j}\right)\right|, \quad d \in \mathbb{N} .
\end{aligned}
$$

The proof of this theorem is essentially based on the following lemma.

Lemma 1 For any $x>0$ and $d \in \mathbb{N}$ the following identities hold:

$$
\begin{aligned}
& \sum_{j=1}^{d} \sum_{\substack{k \in \mathbb{N}: \\
k\left|\ln \omega_{j}\right|>x}}\left(1-\omega_{j}\right) \omega_{j}^{k}=\sum_{\substack{j=1, \ldots, d: \\
\left|\ln \omega_{j}\right|>x}} \omega_{j}+R_{0}(d, x), \\
& \sum_{j=1}^{d} \sum_{\substack{k \in \mathbb{N}: \\
k\left|\ln \omega_{j}\right| \leqslant x}} k\left|\ln \omega_{j}\right|\left(1-\omega_{j}\right) \omega_{j}^{k}=\sum_{\substack{j=1, \ldots, d: \\
\left|\ln \omega_{j}\right| \leqslant x}} \frac{\left|\ln \omega_{j}\right| \omega_{j}}{1-\omega_{j}}-R_{1}(d, x), \\
& \sum_{j=1}^{d}\left[\sum_{\substack{k \in \mathbb{N}: \\
k\left|\ln \omega_{j}\right| \leqslant x}} k^{2}\left|\ln \omega_{j}\right|^{2}\left(1-\omega_{j}\right) \omega_{j}^{k}-\left(\sum_{\substack{k \in \mathbb{N}: \\
k\left|\ln \omega_{j}\right| \leqslant x}} k\left|\ln \omega_{j}\right|\left(1-\omega_{j}\right) \omega_{j}^{k}\right)^{2}\right] \\
& =\sum_{\substack{j=1, \ldots, d: \\
\left|\ln \omega_{j}\right| \leqslant x}} \frac{\left|\ln \omega_{j}\right|^{2} \omega_{j}}{\left(1-\omega_{j}\right)^{2}}-R_{2}(d, x),
\end{aligned}
$$

where

$$
k_{j}(x)=\min \left\{k \in \mathbb{N}: k \geqslant 2, k\left|\ln \omega_{j}\right|>x\right\}
$$


and

$$
\begin{aligned}
R_{0}(d, x):= & \sum_{\substack{j=1, \ldots, d: \\
\left|\ln \omega_{j}\right| \leqslant x}} \omega_{j}^{k_{j}(x)}, \\
R_{1}(d, x):= & \sum_{\substack{j=1, \ldots, d: \\
\left|\ln \omega_{j}\right| \leqslant x}} \frac{\left|\ln \omega_{j}\right| \omega_{j}^{k_{j}(x)}}{1-\omega_{j}}\left(k_{j}(x)\left(1-\omega_{j}\right)+\omega_{j}\right), \\
R_{2}(d, x):= & \sum_{\substack{j=1, \ldots, d: \\
\left|\ln \omega_{j}\right| \leqslant x}} \frac{\left|\ln \omega_{j}\right|^{2} \omega_{j}^{k_{j}(x)}}{\left(1-\omega_{j}\right)^{2}}\left(k_{j}(x)^{2}\left(1-\omega_{j}\right)^{2}\left(1+\omega_{j}^{k_{j}(x)}\right)\right. \\
& \left.\quad+2 k_{j}(x)\left(1-\omega_{j}\right) \omega_{j}^{k_{j}(x)+1}+\left(1-\omega_{j}\right) \omega_{j}+\omega_{j}^{k_{j}(x)+2}\right) .
\end{aligned}
$$

Proof of Lemma 1. We fix $x>0$ and $d \in \mathbb{N}$.

1. Let us prove the first identity. Observe that

$$
\sum_{j=1}^{d} \sum_{\substack{k \in \mathbb{N}: \\ k\left|\ln \omega_{j}\right|>x}}\left(1-\omega_{j}\right) \omega_{j}^{k}=\sum_{\substack{j=1, \ldots, d: \\\left|\ln \omega_{j}\right|>x}} \sum_{\substack{k \in \mathbb{N}: \\ k\left|\ln \omega_{j}\right|>x}}\left(1-\omega_{j}\right) \omega_{j}^{k}+\sum_{\substack{j=1, \ldots, d: \\\left|\ln \omega_{j}\right| \leqslant x}} \sum_{\substack{k \in \mathbb{N}: \\ k\left|\ln \omega_{j}\right|>x}}\left(1-\omega_{j}\right) \omega_{j}^{k} .
$$

Here we have

$$
\sum_{\substack{j=1, \ldots, d: \\\left|\ln \omega_{j}\right|>x}} \sum_{\substack{k \in \mathbb{N}: \\ k\left|\ln \omega_{j}\right|>x}}\left(1-\omega_{j}\right) \omega_{j}^{k}=\sum_{\substack{j=1, \ldots, d: k \in \mathbb{N} \\\left|\ln \omega_{j}\right|>x}} \sum_{k}\left(1-\omega_{j}\right) \omega_{j}^{k}=\sum_{\substack{j=1, \ldots, d: \\\left|\ln \omega_{j}\right|>x}} \omega_{j}
$$

and

$$
\begin{aligned}
\sum_{\substack{j=1, \ldots, d: \\
\left|\ln \omega_{j}\right| \leqslant x}} \sum_{\substack{k \in \mathbb{N}: \\
k\left|\ln \omega_{j}\right|>x}}\left(1-\omega_{j}\right) \omega_{j}^{k} & =\sum_{\substack{j=1, \ldots, d: k \in \mathbb{N}: k \geqslant 2,\left|\ln \omega_{j}\right| \leqslant x}} \sum_{k\left|\ln \omega_{j}\right|>x}\left(1-\omega_{j}\right) \omega_{j}^{k} \\
& =\sum_{\substack{j=1, \ldots, d: k=k_{j}(x) \\
\left|\ln \omega_{j}\right| \leqslant x}} \sum_{\substack{j=1, \ldots, d: \\
\left|\ln \omega_{j}\right| \leqslant x}}^{\infty}\left(1-\omega_{j}\right) \omega_{j}^{k}=\sum_{j}^{k_{j}(x)} .
\end{aligned}
$$

2. Let us prove the second identity. It is obvious that if $\left|\ln \omega_{j}\right|>x$ then there are no any $k \in \mathbb{N}$ such that $k\left|\ln \omega_{j}\right| \leqslant x$. Therefore

$$
\begin{aligned}
\sum_{j=1}^{d} \sum_{\substack{k \in \mathbb{N}: \\
k\left|\ln \omega_{j}\right| \leqslant x}} k\left|\ln \omega_{j}\right|\left(1-\omega_{j}\right) \omega_{j}^{k} & =\sum_{\substack{j=1, \ldots, d: \\
\left|\ln \omega_{j}\right| \leqslant x}} \sum_{\substack{k \in \mathbb{N}: \\
k \ln \omega_{j} \mid \leqslant x}} k\left|\ln \omega_{j}\right|\left(1-\omega_{j}\right) \omega_{j}^{k} \\
& =\sum_{\substack{j=1, \ldots, d: \\
\left|\ln \omega_{j}\right| \leqslant x}}\left|\ln \omega_{j}\right|\left(1-\omega_{j}\right) \sum_{\substack{k \in \mathbb{N}: \\
k\left|\ln \omega_{j}\right| \leqslant x}} k \omega_{j}^{k} .
\end{aligned}
$$

Here

$$
\sum_{\substack{k \in \mathbb{N}: \\ k\left|\ln \omega_{j}\right| \leqslant x}} k \omega_{j}^{k}=\sum_{k=1}^{\infty} k \omega_{j}^{k}-\sum_{k=k_{j}(x)}^{\infty} k \omega_{j}^{k}
$$


It is well known that

$$
\sum_{k=1}^{\infty} k \omega_{j}^{k}=\omega_{j} \sum_{k=1}^{\infty} k \omega_{j}^{k-1}=\frac{\omega_{j}}{\left(1-\omega_{j}\right)^{2}} .
$$

Next, using this fact we get

$$
\begin{aligned}
\sum_{k=k_{j}(x)}^{\infty} k \omega_{j}^{k} & =\omega_{j}^{k_{j}(x)} \sum_{k=k_{j}(x)}^{\infty} k \omega_{j}^{k-k_{j}(x)} \\
& =\omega_{j}^{k_{j}(x)} \sum_{k=k_{j}(x)+1}^{\infty}\left(k-k_{j}(x)\right) \omega_{j}^{k-k_{j}(x)}+k_{j}(x) \omega_{j}^{k_{j}(x)} \sum_{k=k_{j}(x)}^{\infty} \omega_{j}^{k-k_{j}(x)} \\
& =\frac{\omega_{j}^{k_{j}(x)+1}}{\left(1-\omega_{j}\right)^{2}}+\frac{k_{j}(x) \omega_{j}^{k_{j}(x)}}{1-\omega_{j}} .
\end{aligned}
$$

Then

$$
\begin{aligned}
\sum_{j=1}^{d} \sum_{\substack{k \in \mathbb{N}_{j}: \leqslant x \\
k\left|\ln \omega_{j}\right| \leqslant x}} k\left|\ln \omega_{j}\right|\left(1-\omega_{j}\right) \omega_{j}^{k} & =\sum_{\substack{j=1, \ldots, d: \\
\left|\ln \omega_{j}\right| \leqslant x}}\left|\ln \omega_{j}\right|\left(1-\omega_{j}\right)\left(\frac{\omega_{j}}{\left(1-\omega_{j}\right)^{2}}-\frac{\omega_{j}^{k_{j}(x)+1}}{\left(1-\omega_{j}\right)^{2}}-\frac{k_{j}(x) \omega_{j}^{k_{j}(x)}}{1-\omega_{j}}\right) \\
& =\sum_{\substack{j=1, \ldots, d: \\
\left|\ln \omega_{j}\right| \leqslant x}} \frac{\left|\ln \omega_{j}\right| \omega_{j}}{1-\omega_{j}}-\sum_{\substack{j=1, \ldots, d: \\
\left|\ln \omega_{j}\right| \leqslant x}}\left(\frac{\left|\ln \omega_{j}\right| \omega_{j}^{k_{j}(x)+1}}{1-\omega_{j}}+k_{j}(x)\left|\ln \omega_{j}\right| \omega_{j}^{k_{j}(x)}\right) \\
& =\sum_{\substack{j=1, \ldots, d: \\
\left|\ln \omega_{j}\right| \leqslant x}} \frac{\left|\ln \omega_{j}\right| \omega_{j}}{1-\omega_{j}}-\sum_{\substack{j=1, \ldots, d: \\
\left|\ln \omega_{j}\right| \leqslant x}} \frac{\left|\ln \omega_{j}\right| \omega_{j}^{k_{j}(x)}}{1-\omega_{j}}\left(\omega_{j}+k_{j}(x)\left(1-\omega_{j}\right)\right) .
\end{aligned}
$$

3. We now prove the third identity. Let us consider the sum

$$
\begin{aligned}
S_{1}:= & \sum_{j=1}^{d} \sum_{\substack{k \in \mathbb{N}: \\
k\left|\ln \omega_{j}\right| \leqslant x}} k^{2}\left|\ln \omega_{j}\right|^{2}\left(1-\omega_{j}\right) \omega_{j}^{k}=\sum_{\substack{j=1, \ldots, d: \\
\left|\ln \omega_{j}\right| \leqslant x}} \sum_{\substack{k \in \mathbb{N}\left|\ln \omega_{j}\right| \leqslant x \\
k=1, \ldots, d:}} k^{2}\left|\ln \omega_{j}\right|^{2}\left(1-\omega_{j}\right) \omega_{j}^{k} \\
= & \sum_{\substack{j=1, \ldots \\
\left|\ln \omega_{j}\right| \leqslant x}}\left|\ln \omega_{j}\right|^{2}\left(1-\omega_{j}\right) \sum_{\substack{k \in \mathbb{N}: \\
k\left|\ln \omega_{j}\right| \leqslant x}} k^{2} \omega_{j}^{k} .
\end{aligned}
$$

Here

$$
\sum_{\substack{k \in \mathbb{N}: \\ k\left|\ln \omega_{j}\right| \leqslant x}} k^{2} \omega_{j}^{k}=\sum_{k=1}^{\infty} k^{2} \omega_{j}^{k}-\sum_{k=k_{j}(x)}^{\infty} k^{2} \omega_{j}^{k}
$$

It is easily seen that

$$
\begin{gathered}
\sum_{k=1}^{\infty} k^{2} \omega_{j}^{k}=\sum_{k=1}^{\infty} k(k-1) \omega_{j}^{k}+\sum_{k=1}^{\infty} k \omega_{j}^{k}=\omega_{j}^{2} \sum_{k=2}^{\infty} k(k-1) \omega_{j}^{k-2}+\sum_{k=1}^{\infty} k \omega_{j}^{k} \\
=\frac{2 \omega_{j}^{2}}{\left(1-\omega_{j}\right)^{3}}+\frac{\omega_{j}}{\left(1-\omega_{j}\right)^{2}}=\frac{\omega_{j}+\omega_{j}^{2}}{\left(1-\omega_{j}\right)^{3}} .
\end{gathered}
$$


Next, we write

$$
\begin{aligned}
\sum_{k=k_{j}(x)}^{\infty} k^{2} \omega_{j}^{k} & =\sum_{k=k_{j}(x)}^{\infty}\left(k-k_{j}(x)\right)^{2} \omega_{j}^{k}+2 k_{j}(x) \sum_{k=k_{j}(x)}^{\infty} k \omega_{j}^{k}-k_{j}(x)^{2} \sum_{k=k_{j}(x)}^{\infty} \omega_{j}^{k} \\
& =\omega_{j}^{k_{j}(x)} \sum_{k=k_{j}(x)+1}^{\infty}\left(k-k_{j}(x)\right)^{2} \omega_{j}^{k-k_{j}(x)}+2 k_{j}(x) \sum_{k=k_{j}(x)}^{\infty} k \omega_{j}^{k}-k_{j}(x)^{2} \sum_{k=k_{j}(x)}^{\infty} \omega_{j}^{k} .
\end{aligned}
$$

Due to above remarks we get

$$
\begin{aligned}
\sum_{k=k_{j}(x)}^{\infty} k^{2} \omega_{j}^{k}= & \omega_{j}^{k_{j}(x)}\left(\frac{2 \omega_{j}^{2}}{\left(1-\omega_{j}\right)^{3}}+\frac{\omega_{j}}{\left(1-\omega_{j}\right)^{2}}\right)+2 k_{j}(x)\left(\frac{\omega_{j}^{k_{j}(x)+1}}{\left(1-\omega_{j}\right)^{2}}+\frac{k_{j}(x) \omega_{j}^{k_{j}(x)}}{1-\omega_{j}}\right) \\
& -k_{j}(x)^{2} \cdot \frac{\omega_{j}^{k_{j}(x)}}{1-\omega_{j}} \\
= & \frac{2 \omega_{j}^{k_{j}(x)+2}}{\left(1-\omega_{j}\right)^{3}}+\frac{\left(2 k_{j}(x)+1\right) \omega_{j}^{k_{j}(x)+1}}{\left(1-\omega_{j}\right)^{2}}+\frac{k_{j}(x)^{2} \omega_{j}^{k_{j}(x)}}{1-\omega_{j}} .
\end{aligned}
$$

Thus we obtain

$$
\begin{aligned}
S_{1} & =\sum_{\substack{j=1, \ldots, d: \\
\left|\ln \omega_{j}\right| \leqslant x}}\left|\ln \omega_{j}\right|^{2}\left(1-\omega_{j}\right)\left(\frac{\omega_{j}+\omega_{j}^{2}}{\left(1-\omega_{j}\right)^{3}}-\frac{2 \omega_{j}^{k_{j}(x)+2}}{\left(1-\omega_{j}\right)^{3}}-\frac{\left(2 k_{j}(x)+1\right) \omega_{j}^{k_{j}(x)+1}}{\left(1-\omega_{j}\right)^{2}}-\frac{k_{j}(x)^{2} \omega_{j}^{k_{j}(x)}}{1-\omega_{j}}\right) \\
& =\sum_{\substack{j=1, \ldots, d: \\
\left|\ln \omega_{j}\right| \leqslant x}}\left|\ln \omega_{j}\right|^{2}\left(\frac{\omega_{j}+\omega_{j}^{2}}{\left(1-\omega_{j}\right)^{2}}-\frac{2 \omega_{j}^{k_{j}(x)+2}}{\left(1-\omega_{j}\right)^{2}}-\frac{\left(2 k_{j}(x)+1\right) \omega_{j}^{k_{j}(x)+1}}{1-\omega_{j}}-k_{j}(x)^{2} \omega_{j}^{k_{j}(x)}\right) .
\end{aligned}
$$

We now consider the sums

$$
\begin{aligned}
S_{2} & :=\sum_{j=1}^{d}\left(\sum_{\substack{k \in \mathbb{N}: \\
k\left|\ln \omega_{j}\right| \leqslant x}} k\left|\ln \omega_{j}\right|\left(1-\omega_{j}\right) \omega_{j}^{k}\right)^{2} \\
& =\sum_{\substack{j=1, \ldots, d: \\
\left|\ln \omega_{j}\right| \leqslant x}}\left(\sum_{\substack{k \in \mathbb{N}: \\
k\left|\ln \omega_{j}\right| \leqslant x}} k\left|\ln \omega_{j}\right|\left(1-\omega_{j}\right) \omega_{j}^{k}\right)^{2} \\
& =\sum_{\substack{j=1, \ldots, d: \\
\left|\ln \omega_{j}\right| \leqslant x}}\left|\ln \omega_{j}\right|^{2}\left(1-\omega_{j}\right)^{2}\left(\sum_{k=1}^{\infty} k \omega_{j}^{k}-\sum_{k=k_{j}(x)}^{\infty} k \omega_{j}^{k}\right)^{2} .
\end{aligned}
$$

By the above remarks, we have

$$
\begin{aligned}
\left(\sum_{k=1}^{\infty} k \omega_{j}^{k}-\sum_{k=k_{j}(x)}^{\infty} k \omega_{j}^{k}\right)^{2}= & \left(\frac{\omega_{j}}{\left(1-\omega_{j}\right)^{2}}-\frac{\omega_{j}^{k_{j}(x)+1}}{\left(1-\omega_{j}\right)^{2}}-\frac{k_{j}(x) \omega_{j}^{k_{j}(x)}}{1-\omega_{j}}\right)^{2} \\
= & \frac{\omega_{j}^{2}}{\left(1-\omega_{j}\right)^{4}}+\frac{\omega_{j}^{2 k_{j}(x)+2}}{\left(1-\omega_{j}\right)^{4}}+\frac{k_{j}(x)^{2} \omega_{j}^{2 k_{j}(x)}}{\left(1-\omega_{j}\right)^{2}}-\frac{2 \omega_{j}^{k_{j}(x)+2}}{\left(1-\omega_{j}\right)^{4}} \\
& -\frac{2 k_{j}(x) \omega_{j}^{k_{j}(x)+1}}{\left(1-\omega_{j}\right)^{3}}+\frac{2 k_{j}(x) \omega_{j}^{2 k_{j}(x)+1}}{\left(1-\omega_{j}\right)^{3}} .
\end{aligned}
$$


Hence we get

$$
\begin{aligned}
S_{2}= & \sum_{\substack{j=1, \ldots, d: \\
\left|\ln \omega_{j}\right| \leqslant x}}\left|\ln \omega_{j}\right|^{2}\left(\frac{\omega_{j}^{2}}{\left(1-\omega_{j}\right)^{2}}+\frac{\omega_{j}^{2 k_{j}(x)+2}}{\left(1-\omega_{j}\right)^{2}}+k_{j}(x)^{2} \omega_{j}^{2 k_{j}(x)}-\frac{2 \omega_{j}^{k_{j}(x)+2}}{\left(1-\omega_{j}\right)^{2}}\right. \\
& \left.-\frac{2 k_{j}(x) \omega_{j}^{k_{j}(x)+1}}{1-\omega_{j}}+\frac{2 k_{j}(x) \omega_{j}^{2 k_{j}(x)+1}}{1-\omega_{j}}\right) .
\end{aligned}
$$

Thus we have

$$
\begin{aligned}
S_{1}-S_{2}= & \sum_{\substack{j=1, \ldots, d: \\
\left|\ln \omega_{j}\right| \leqslant x}}\left|\ln \omega_{j}\right|^{2}\left(\frac{\omega_{j}}{\left(1-\omega_{j}\right)^{2}}-\frac{\omega_{j}^{k_{j}(x)+1}}{1-\omega_{j}}-k_{j}(x)^{2} \omega_{j}^{k_{j}(x)}\right. \\
& \left.-\frac{\omega_{j}^{2 k_{j}(x)+2}}{\left(1-\omega_{j}\right)^{2}}-k_{j}(x)^{2} \omega_{j}^{2 k_{j}(x)}-\frac{2 k_{j}(x) \omega_{j}^{2 k_{j}(x)+1}}{1-\omega_{j}}\right) \\
= & \sum_{\substack{j=1, \ldots, d: \\
\left|\ln \omega_{j}\right| \leqslant x}} \frac{\left|\ln \omega_{j}\right|^{2} \omega_{j}}{\left(1-\omega_{j}\right)^{2}}-\sum_{\substack{j=1, \ldots, d: \\
\left|\ln \omega_{j}\right| \leqslant x}} \frac{\left|\ln \omega_{j}\right|^{2} \omega_{j}^{k_{j}(x)}}{\left(1-\omega_{j}\right)^{2}}\left(\left(1-\omega_{j}\right) \omega_{j}+k_{j}(x)^{2}\left(1-\omega_{j}\right)^{2}\right. \\
& \left.+\omega_{j}^{k_{j}(x)+2}+k_{j}(x)^{2}\left(1-\omega_{j}\right)^{2} \omega_{j}^{k_{j}(x)}+2 k_{j}(x)\left(1-\omega_{j}\right) \omega_{j}^{k_{j}(x)+1}\right) \\
= & \sum_{\substack{j=1, \ldots, d: \\
\left|\ln \omega_{j}\right| \leqslant x}} \frac{\left|\ln \omega_{j}\right|^{2} \omega_{j}}{\left(1-\omega_{j}\right)^{2}}-\sum_{\substack{j=1, \ldots, d: \\
\left|\ln \omega_{j}\right| \leqslant x}} \frac{\left|\ln \omega_{j}\right|^{2} \omega_{j}^{k_{j}(x)}}{\left(1-\omega_{j}\right)^{2}}\left(k_{j}(x)^{2}\left(1-\omega_{j}\right)^{2}\left(1+\omega_{j}^{k_{j}(x)}\right)\right. \\
& \left.+2 k_{j}(x)\left(1-\omega_{j}\right) \omega_{j}^{k_{j}(x)+1}+\left(1-\omega_{j}\right) \omega_{j}+\omega_{j}^{k_{j}(x)+2}\right) .
\end{aligned}
$$

So we obtain the third identity.

We now turn to proof of the theorem.

Proof of Theorem 2, We first recall that $\mathcal{C}(q)=(0,1)$, which follows from properties of selfdecomposable distribution functions (see [11], Remarks 2 and 8). Thus, by Theorem 1 from [11, the condition (13) is equivalent to the convergence (91), where $G$ is the given self-decomposable law with the triplet $(c, v, L)$ and $G_{d}, d \in \mathbb{N}$, are defined by (10) for given $a_{d}, b_{d}$, and $Y_{d}, d \in \mathbb{N}$. According to (11), $G_{d}, d \in \mathbb{N}$, are distribution functions of centered and normalized sums of nonnegative independent random variables $\hat{U}_{j}, j \in \mathbb{N}$, satisfying (12). Hence for the convergence (9) the conditions $\left(\mathrm{A}_{1}\right),(\mathrm{B}),(\mathrm{C})$ of Theorem 11 from [11] (Appendix) are necessary and sufficient (with $Y_{j}:=\hat{U}_{j}, j \in \mathbb{N}, A_{d}:=\hat{a}_{d}, B_{d}:=b_{d}, \gamma:=c$, and $\left.\sigma^{2}:=v\right)$. Thus (13) is equivalent to the following ensemble of conditions:

$$
\lim _{d \rightarrow \infty} \sum_{j=1}^{d} \mathbb{P}\left(\hat{U}_{j}>\tau b_{d}\right)=-L(\tau) \quad \text { for all } \quad \tau>0
$$




$$
\begin{aligned}
& \lim _{d \rightarrow \infty} \frac{1}{b_{d}}\left(\sum_{j=1}^{d} \mathbb{E}\left[\hat{U}_{j} \mathbb{1}\left(\left|\hat{U}_{j}\right| \leqslant \tau b_{d}\right)\right]-\hat{a}_{d}\right)=c+\gamma_{\tau} \text { for all } \tau>0 ; \\
& \lim _{\tau \rightarrow 0} \frac{\lim _{d \rightarrow \infty}}{b_{d}^{2}} \sum_{j=1}^{d} \operatorname{Var}\left[\hat{U}_{j} \mathbb{1}\left(\left|\hat{U}_{j}\right| \leqslant \tau b_{d}\right)\right]=\lim _{\tau \rightarrow 0} \varlimsup_{d \rightarrow \infty} \frac{1}{b_{d}^{2}} \sum_{j=1}^{d} \operatorname{Var}\left[\hat{U}_{j} \mathbb{1}\left(\left|\hat{U}_{j}\right| \leqslant \tau b_{d}\right)\right]=v .
\end{aligned}
$$

Here $\gamma_{\tau}$ is defined by (14).

Let us write the sums in these conditions in terms of $\omega_{j}$. First, note that

$$
\begin{aligned}
\mathbb{P}\left(\hat{U}_{j}>\tau b_{d}\right) & =\sum_{\substack{k \in \mathbb{N}: \\
k\left|\ln \omega_{j}\right|>\tau b_{d}}}\left(1-\omega_{j}\right) \omega_{j}^{k}, \\
\mathbb{E}\left[\hat{U}_{j} \mathbb{1}\left(\left|\hat{U}_{j}\right| \leqslant \tau b_{d}\right)\right] & =\sum_{\substack{k \in \mathbb{N}: \\
k\left|\ln \omega_{j}\right| \leqslant \tau b_{d}}} k\left|\ln \omega_{j}\right|\left(1-\omega_{j}\right) \omega_{j}^{k}, \\
\operatorname{Var}\left[\hat{U}_{j} \mathbb{1}\left(\left|\hat{U}_{j}\right| \leqslant \tau b_{d}\right)\right] & =\mathbb{E}\left[\hat{U}_{j}^{2} \mathbb{1}\left(\left|\hat{U}_{j}\right| \leqslant \tau b_{d}\right)\right]-\left(\mathbb{E}\left[\hat{U}_{j} \mathbb{1}\left(\left|\hat{U}_{j}\right| \leqslant \tau b_{d}\right)\right]\right)^{2} \\
& =\sum_{\substack{k \in \mathbb{N}: \\
k\left|\ln \omega_{j}\right| \leqslant \tau b_{d}}} k^{2}\left|\ln \omega_{j}\right|^{2}\left(1-\omega_{j}\right) \omega_{j}^{k}-\left(\sum_{\substack{k \in \mathbb{N}: \\
k\left|\ln \omega_{j}\right| \leqslant \tau b_{d}}} k\left|\ln \omega_{j}\right|\left(1-\omega_{j}\right) \omega_{j}^{k}\right)^{2} .
\end{aligned}
$$

Next, applying Lemma 1 we have

$$
\begin{aligned}
\sum_{j=1}^{d} \mathbb{P}\left(\hat{U}_{j}>\tau b_{d}\right) & =\sum_{\substack{j=1, \ldots, d \\
\left|\ln \omega_{j}\right|>\tau b_{d}}} \omega_{j}+R_{0}\left(d, \tau b_{d}\right), \\
\sum_{j=1}^{d} \mathbb{E}\left[\hat{U}_{j} \mathbb{1}\left(\left|\hat{U}_{j}\right| \leqslant \tau b_{d}\right)\right] & =\sum_{\substack{j=1, \ldots, d: \\
\left|\ln \omega_{j}\right| \leqslant \tau b_{d}}} \frac{\left|\ln \omega_{j}\right| \omega_{j}}{1-\omega_{j}}-R_{1}\left(d, \tau b_{d}\right), \\
\sum_{j=1}^{d} \operatorname{Var}\left[\hat{U}_{j} \mathbb{1}\left(\left|\hat{U}_{j}\right| \leqslant \tau b_{d}\right)\right] & =\sum_{\substack{j=1, \ldots, d: \\
\left|\ln \omega_{j}\right| \leqslant \tau b_{d}}} \frac{\left|\ln \omega_{j}\right|^{2} \omega_{j}}{\left(1-\omega_{j}\right)^{2}}-R_{2}\left(d, \tau b_{d}\right),
\end{aligned}
$$

where the functions $R_{0}, R_{1}$, and $R_{2}$ are defined as in Lemma 1. Therefore (13) is equivalent to the following three conditions

$\left(\mathrm{A}^{\prime}\right) \quad \lim _{d \rightarrow \infty}\left(\sum_{\substack{j=1, \ldots, d: \\\left|\ln \omega_{j}\right|>\tau b_{d}}} \omega_{j}+R_{0}\left(d, \tau b_{d}\right)\right)=-L(\tau)$ for all $\tau>0 ;$

$$
\lim _{d \rightarrow \infty} \frac{1}{b_{d}}\left(\sum_{\substack{j=1, \ldots, d: \\\left|\ln \omega_{j}\right| \leqslant \tau b_{d}}} \frac{\left|\ln \omega_{j}\right| \omega_{j}}{1-\omega_{j}}-R_{1}\left(d, \tau b_{d}\right)-\hat{a}_{d}\right)=c+\gamma_{\tau} \quad \text { for all } \quad \tau>0
$$




$$
\begin{aligned}
\left(\mathrm{C}^{\prime}\right) \quad \lim _{\tau \rightarrow 0} \frac{\lim }{d \rightarrow \infty} \frac{1}{b_{d}^{2}}\left(\sum_{\substack{j=1, \ldots, d: \\
\left|\ln \omega_{j}\right| \leqslant \tau b_{d}}} \frac{\left|\ln \omega_{j}\right|^{2} \omega_{j}}{\left(1-\omega_{j}\right)^{2}}-R_{2}\left(d, \tau b_{d}\right)\right)= \\
\quad=\lim _{\tau \rightarrow 0} \varlimsup_{\substack{d \rightarrow \infty \\
d \rightarrow \infty}} \frac{1}{b_{d}^{2}}\left(\sum_{\substack{j=1, \ldots, d: \\
\left|\ln \omega_{j}\right| \leqslant \tau b_{d}}} \frac{\left|\ln \omega_{j}\right|^{2} \omega_{j}}{\left(1-\omega_{j}\right)^{2}}-R_{2}\left(d, \tau b_{d}\right)\right)=v^{2} .
\end{aligned}
$$

We first show that $(\mathrm{A}),(\mathrm{B}),(\mathrm{C})$ imply $\left(\mathrm{A}^{\prime}\right),\left(\mathrm{B}^{\prime}\right),\left(\mathrm{C}^{\prime}\right)$. Due to the condition $(\mathrm{C})$, there exist $\tau_{0}>0$ and $C_{0}>0$ such that for all $d \in \mathbb{N}$ we have

$$
\frac{1}{b_{d}^{2}} \sum_{\substack{j=1, \ldots, d: \\\left|\ln \omega_{j}\right| \leqslant \tau_{0} b_{d}}} \frac{\left|\ln \omega_{j}\right|^{2} \omega_{j}}{\left(1-\omega_{j}\right)^{2}} \leqslant C_{0}
$$

Since $\omega_{j} \in(0,1)$, the inequality $\left|\ln \omega_{j}\right|>1-\omega_{j}$ always holds. Hence we conclude that

$$
\frac{1}{b_{d}^{2}} \sum_{\substack{j=1, \ldots, d: \\\left|\ln \omega_{j}\right| \leqslant \tau_{0} b_{d}}} \omega_{j} \leqslant C_{0}, \quad d \in \mathbb{N}
$$

We first show that $R_{0}\left(d, \tau b_{d}\right) \rightarrow 0, d \rightarrow \infty$, for every $\tau>0$. We fix $\tau>0$ and $\tau_{*}<\min \left\{\tau_{0}, \tau\right\}$. Observe that

$$
R_{0}\left(d, \tau b_{d}\right)=\sum_{\substack{j=1, \ldots, d: \\\left|\ln \omega_{j}\right| \leqslant \tau b_{d}}} \omega_{j}^{k_{j}\left(\tau b_{d}\right)} \leqslant \sum_{\substack{j=1, \ldots, d: \\\left|\ln \omega_{j}\right| \leqslant \tau_{*} b_{d}}} \omega_{j}^{k_{j}\left(\tau b_{d}\right)}+\sum_{\substack{j=1, \ldots, d: \\\left|\ln \omega_{j}\right|>\tau_{*} b_{d}}} \omega_{j}^{k_{j}\left(\tau b_{d}\right)}
$$

By the definition of $k_{j}(\cdot)$ (see Lemma 1), we have the inequalities $k_{j}\left(\tau b_{d}\right) \geqslant 2$ and $\omega_{j}^{k_{j}\left(\tau b_{d}\right)} \leqslant e^{-\tau b_{d}}$, which give

$$
R_{0}\left(d, \tau b_{d}\right) \leqslant e^{-\tau b_{d}} \sum_{\substack{j=1, \ldots, d: \\\left|\ln \omega_{j}\right| \leqslant \tau_{*} b_{d}}} 1+\sum_{\substack{j=1, \ldots, d: \\\left|\ln \omega_{j}\right|>\tau_{*} b_{d}}} \omega_{j}^{2}
$$

Using conditions in the sums, we obtain

$$
\begin{aligned}
R_{0}\left(d, \tau b_{d}\right) & \leqslant e^{-\left(\tau-\tau_{*}\right) b_{d}} \sum_{\substack{j=1, \ldots, d: \\
\left|\ln \omega_{j}\right| \leqslant \tau_{*} b_{d}}} \omega_{j}+e^{-\tau_{*} b_{d}} \sum_{\substack{j=1, \ldots, d: \\
\left|\ln \omega_{j}\right|>\tau_{*} b_{d}}} \omega_{j} \\
& \leqslant e^{-\left(\tau-\tau_{*}\right) b_{d}} \sum_{\substack{j=1, \ldots, d: \\
\left|\ln \omega_{j}\right| \leqslant \tau_{0} b_{d}}} \omega_{j}+e^{-\tau_{*} b_{d}} \sum_{\substack{j=1, \ldots, d: \\
\left|\ln \omega_{j}\right|>\tau_{*} b_{d}}} \omega_{j} .
\end{aligned}
$$

Here the first sum is less than $C_{0} b_{d}^{2}$ by (17) and the second sum is bounded by some constant $C_{1}$ due to (A). Thus

$$
R_{0}\left(d, \tau b_{d}\right) \leqslant C_{0} b_{d}^{2} e^{-\left(\tau-\tau_{*}\right) b_{d}}+C_{1} e^{-\tau_{*} b_{d}}
$$

Since $\tau>\tau_{*}>0$ and $b_{d} \rightarrow \infty$, we obtain that $R_{0}\left(d, \tau b_{d}\right) \rightarrow 0, d \rightarrow \infty$. This, together with (A), yields $\left(\mathrm{A}^{\prime}\right)$. 
We now consider

$$
R_{1}\left(d, \tau b_{d}\right)=\sum_{\substack{j=1, \ldots, d: \\\left|\ln \omega_{j}\right| \leqslant \tau b_{d}}} \frac{\left|\ln \omega_{j}\right| \omega_{j}^{k_{j}\left(\tau b_{d}\right)}}{1-\omega_{j}}\left(k_{j}\left(\tau b_{d}\right)\left(1-\omega_{j}\right)+\omega_{j}\right) .
$$

According to the definition of $k_{j}(\cdot)$, we have

$$
k_{j}\left(\tau b_{d}\right) \leqslant 2\left(k_{j}\left(\tau b_{d}\right)-1\right) \leqslant \frac{2 \tau b_{d}}{\left|\ln \omega_{j}\right|} .
$$

Hence

$$
R_{1}\left(d, \tau b_{d}\right) \leqslant 2 \tau b_{d} \sum_{\substack{j=1, \ldots, d: \\\left|\ln \omega_{j}\right| \leqslant \tau b_{d}}} \omega_{j}^{k_{j}\left(\tau b_{d}\right)}+\sum_{\substack{j=1, \ldots, d: \\\left|\ln \omega_{j}\right| \leqslant \tau b_{d}}} \frac{\left|\ln \omega_{j}\right| \omega_{j}}{1-\omega_{j}} \cdot \omega_{j}^{k_{j}\left(\tau b_{d}\right)}
$$

Note that the function $\omega_{j} \mapsto \frac{\left|\ln \omega_{j}\right| \omega_{j}}{1-\omega_{j}}$ is bounded by some constant $C_{3}$ for all values $\omega_{j} \in(0,1)$. Therefore

$$
R_{1}(d, \tau d) \leqslant\left(2 \tau b_{d}+C_{3}\right) \sum_{\substack{j=1, \ldots, d: \\\left|\ln \omega_{j}\right| \leqslant \tau b_{d}}} \omega_{j}^{k_{j}\left(\tau b_{d}\right)}=\left(2 \tau b_{d}+C_{3}\right) \cdot R_{0}\left(d, \tau b_{d}\right) .
$$

Since $R_{0}\left(d, \tau b_{d}\right) \rightarrow 0, d \rightarrow \infty$, we have $R_{1}(d, \tau d)=o\left(b_{d}\right), d \rightarrow \infty$. Due to $(\mathrm{B})$, this yields $\left(\mathrm{B}^{\prime}\right)$.

We now consider

$$
\begin{aligned}
R_{2}\left(d, \tau b_{d}\right)= & \sum_{\substack{j=1, \ldots, d: \\
\left|\ln \omega_{j}\right| \leqslant \tau b_{d}}} \frac{\left|\ln \omega_{j}\right|^{2} \omega_{j}^{k_{j}\left(\tau b_{d}\right)}}{\left(1-\omega_{j}\right)^{2}}\left(k_{j}\left(\tau b_{d}\right)^{2}\left(1-\omega_{j}\right)^{2}\left(1+\omega_{j}^{k_{j}\left(\tau b_{d}\right)}\right)\right. \\
& \left.\quad+2 k_{j}\left(\tau b_{d}\right)\left(1-\omega_{j}\right) \omega_{j}^{k_{j}\left(\tau b_{d}\right)+1}+\omega_{j}^{k_{j}\left(\tau b_{d}\right)+2}+\left(1-\omega_{j}\right) \omega_{j}\right) .
\end{aligned}
$$

Using the inequality (18),

$$
\begin{aligned}
R_{2}\left(d, \tau b_{d}\right) \leqslant & \left(2 \tau b_{d}\right)^{2} \sum_{\substack{j=1, \ldots, d: \\
\left|\ln \omega_{j}\right| \leqslant \tau b_{d}}} \omega_{j}^{k_{j}\left(\tau b_{d}\right)}\left(1+\omega_{j}^{k_{j}\left(\tau b_{d}\right)}\right)+4 \tau b_{d} \sum_{\substack{j=1, \ldots, d: \\
\left|\ln \omega_{j}\right| \leqslant \tau b_{d}}} \frac{\left|\ln \omega_{j}\right|}{1-\omega_{j}} \omega_{j}^{2 k_{j}\left(\tau b_{d}\right)+1} \\
& +\sum_{\substack{j=1, \ldots, d: \\
\left|\ln \omega_{j}\right| \leqslant \tau b_{d}}} \frac{\left|\ln \omega_{j}\right|^{2} \omega_{j}^{2 k_{j}\left(\tau b_{d}\right)+2}}{\left(1-\omega_{j}\right)^{2}}+\sum_{\substack{j=1, \ldots, d: \\
\left|\ln \omega_{j}\right| \leqslant \tau b_{d}}} \frac{\left|\ln \omega_{j}\right|^{2} \omega_{j}^{k_{j}\left(\tau b_{d}\right)+1}}{1-\omega_{j}} .
\end{aligned}
$$

The function $\omega_{j} \mapsto \frac{\left|\ln \omega_{j}\right|^{2} \omega_{j}}{1-\omega_{j}}$ is bounded by some constant $C_{4}$ for all values $\omega_{j} \in(0,1)$. Using this and the same fact about $\omega_{j} \mapsto \frac{\left|\ln \omega_{j}\right| \omega_{j}}{1-\omega_{j}}$, we obtain

$$
\begin{aligned}
R_{2}\left(d, \tau b_{d}\right) \leqslant & 4 \tau^{2} b_{d}^{2} \sum_{\substack{j=1, \ldots, d: \\
\left|\ln \omega_{j}\right| \leqslant \tau b_{d}}} \omega_{j}^{k_{j}\left(\tau b_{d}\right)}\left(1+\omega_{j}^{k_{j}\left(\tau b_{d}\right)}\right)+4 \tau b_{d} \sum_{\substack{j=1, \ldots, d: \\
\left|\ln \omega_{j}\right| \leqslant \tau b_{d}}} \omega_{j}^{2 k_{j}\left(\tau b_{d}\right)} \\
& +C_{3}^{2} \sum_{\substack{j=1, \ldots, d: \\
\left|\ln \omega_{j}\right| \leqslant \tau b_{d}}} \omega_{j}^{k_{j}\left(\tau b_{d}\right)}+C_{4} \sum_{\substack{j=1, \ldots, d: \\
\left|\ln \omega_{j}\right| \leqslant \tau b_{d}}} \omega_{j}^{2 k_{j}\left(\tau b_{d}\right)} .
\end{aligned}
$$


Next, since $\omega_{j}^{k_{j}\left(\tau b_{d}\right)} \leqslant 1$, we have

$$
\begin{aligned}
R_{2}\left(d, \tau b_{d}\right) & \leqslant\left(8 \tau^{2} b_{d}^{2}+4 \tau b_{d}+C_{3}^{2}+C_{4}\right) \sum_{\substack{j=1, \ldots, d: \\
\left|\ln \omega_{j}\right| \leqslant \tau b_{d}}} \omega_{j}^{k_{j}\left(\tau b_{d}\right)} \\
& =\left(8 \tau^{2} b_{d}^{2}+4 \tau b_{d}+C_{3}^{2}+C_{4}\right) R_{0}\left(d, \tau b_{d}\right) .
\end{aligned}
$$

Here $R_{0}\left(d, \tau b_{d}\right) \rightarrow 0, d \rightarrow \infty$. Hence $R_{2}\left(d, \tau b_{d}\right)=o\left(b_{d}^{2}\right), d \rightarrow \infty$. Due to $(\mathrm{C})$, this yields $\left(\mathrm{C}^{\prime}\right)$.

We now show that $\left(\mathrm{A}^{\prime}\right),\left(\mathrm{B}^{\prime}\right),\left(\mathrm{C}^{\prime}\right)$ imply $(\mathrm{A}),(\mathrm{B}),(\mathrm{C})$. From $\left(\mathrm{A}^{\prime}\right)$ it follows that $\sup _{d \in \mathbb{N}} R_{0}\left(d, \tau b_{d}\right)<$ $\infty$ for every $\tau>0$. By this relation and (20), we have

$$
\sup _{d \in \mathbb{N}} \frac{1}{b_{d}^{2}} R_{2}\left(d, \tau b_{d}\right)<\infty \quad \text { for every } \tau>0 .
$$

This and $\left(\mathrm{C}^{\prime}\right)$ yield (16) for every $d \in \mathbb{N}$ and some $\tau_{0}>0$ and $C_{0}>0$. Hence, as we showed above, it follows that $R_{0}\left(d, \tau b_{d}\right) \rightarrow 0, d \rightarrow \infty$, for every $\tau>0$. Here we use

$$
\sup _{d \in \mathbb{N}} \sum_{\substack{j=1, \ldots, d: \\\left|\ln \omega_{j}\right|>\tau b_{d}}} \omega_{j}<\infty \quad \text { for every } \tau>0,
$$

which follows from $\left(\mathrm{A}^{\prime}\right)$. Next, from (19) and (20) we correspondingly obtain that $R_{1}\left(d, \tau b_{d}\right)=o\left(b_{d}\right)$ and $R_{2}\left(d, \tau b_{d}\right)=o\left(b_{d}^{2}\right), d \rightarrow \infty$, for every $\tau>0$. These relations for $R_{k}\left(d, \tau b_{d}\right), k=1,2,3$, and conditions $\left(\mathrm{A}^{\prime}\right),\left(\mathrm{B}^{\prime}\right),\left(\mathrm{C}^{\prime}\right)$ give $(\mathrm{A}),(\mathrm{B}),(\mathrm{C})$.

\section{$4 \quad$ Examples}

In this section we will study asymptotics for $n^{Y_{d}}(\varepsilon)$ under particular additional assumptions on the sequence of the length scale parameters $\sigma_{j}, j \in \mathbb{N}$.

We begin with the case, when the sequence $\left(\sigma_{j}\right)_{j \in \mathbb{N}}$ tends to to a non-negative constant $\sigma$. Observe that here approximation complexity $n^{Y_{d}}(\varepsilon)$ is unbounded due to Proposition 2. The following proposition specifies asymptotic (13) for this case.

Proposition 3 Suppose that $\sigma_{j} \rightarrow \sigma, 0 \leqslant \sigma<\infty$. Then

$$
\forall \varepsilon \in(0,1) \quad \ln n^{Y_{d}}(\varepsilon)=a_{d}+\Phi^{-1}\left(1-\varepsilon^{2}\right) b_{d}+o\left(b_{d}\right), \quad d \rightarrow \infty,
$$

where $\Phi$ is the distribution function of the standard Gaussian law,

$$
\begin{gathered}
a_{d}=\sum_{j=1}^{d}\left(\frac{\left|\ln \omega_{j}\right| \omega_{j}}{1-\omega_{j}}+\left|\ln \left(1-\omega_{j}\right)\right|\right), \quad b_{d}=\rho \sqrt{d}, \quad d \in \mathbb{N}, \\
\rho=\lim _{j \rightarrow \infty} \frac{\left|\ln \omega_{j}\right| \sqrt{\omega_{j}}}{1-\omega_{j}}=\left\{\begin{array}{ll}
\frac{|\ln \omega| \sqrt{\omega}}{1-\omega}, & \sigma>0, \\
1, & \sigma=0,
\end{array} \quad \omega=\lim _{j \rightarrow \infty} \omega_{j}=\left(1+\frac{\sigma^{2}}{2}\left(1+\sqrt{1+\frac{4}{\sigma^{2}}}\right)\right)^{-1}\right.
\end{gathered}
$$

Remark 1 If $\sigma>0$ then

$$
a_{d}=\left(\frac{|\ln \omega| \omega}{1-\omega}+|\ln (1-\omega)|\right) d+o(d), \quad d \rightarrow \infty
$$


Indeed, since $\lim _{j \rightarrow \infty} \omega_{j}=\omega$ we have

$$
a_{d} \sim d \cdot \lim _{d \rightarrow \infty}\left(\frac{\left|\ln \omega_{d}\right| \omega_{d}}{1-\omega_{d}}+\left|\ln \left(1-\omega_{d}\right)\right|\right)=d \cdot\left(\frac{|\ln \omega| \omega}{1-\omega}+|\ln (1-\omega)|\right), \quad d \rightarrow \infty
$$

Remark 2 If $\sigma=0$ then $a_{d}$ can increase arbitrarily fast.

It is seen from the inequality

$$
a_{d} \geqslant\left|\ln \left(1-\omega_{d}\right)\right|, \quad d \in \mathbb{N}
$$

and from the relation $\lim _{d \rightarrow \infty} \omega_{d}=1$, which holds under the assumption $\sigma=0$.

More sharp asymptotics of $a_{d}\left(\right.$ up to $\left.o\left(b_{d}\right)\right)$ require additional assumptions on the rate of convergence of $\sigma_{j}$ to $\sigma$ as $j \rightarrow \infty$.

Proof of Proposition 3. It is well known that $\Phi$ is a self-decomposable distribution function with the triplet $(0,1, L)$, where $L(x)=0$ for all $x \in \mathbb{R} \backslash\{0\}$. By Theorem 2 it is sufficient to check the following ensemble of conditions to obtain the asymptotics (21):

$$
\begin{aligned}
& \lim _{d \rightarrow \infty} \sum_{\substack{j=1, \ldots, d \\
\left|\ln \omega_{j}\right|>\tau b_{d}}} \omega_{j}=0, \quad \tau>0 \\
& \lim _{d \rightarrow \infty} \frac{1}{b_{d}}\left(\sum_{\substack{j=1, \ldots, d \\
\left|\ln \omega_{j}\right| \leqslant \tau b_{d}}} \frac{\left|\ln \omega_{j}\right| \omega_{j}}{1-\omega_{j}}-\hat{a}_{d}\right)=0, \quad \tau>0 ; \\
& \lim _{\tau \rightarrow 0} \frac{\lim }{d \rightarrow \infty} \frac{1}{b_{d}^{2}} \sum_{\substack{j=1, \ldots, d \\
\left|\ln \omega_{j}\right| \leqslant \tau b_{d}}} \frac{\left|\ln \omega_{j}\right|^{2} \omega_{j}}{\left(1-\omega_{j}\right)^{2}}=\lim _{\tau \rightarrow 0} \varlimsup_{\substack{d \rightarrow \infty \\
d \rightarrow \infty}} \frac{1}{b_{d}^{2}} \sum_{\substack{j=1, \ldots, d \\
\left|\ln \omega_{j}\right| \leqslant \tau b_{d}}} \frac{\left|\ln \omega_{j}\right|^{2} \omega_{j}}{\left(1-\omega_{j}\right)^{2}}=1 .
\end{aligned}
$$

Here $\hat{a}_{d}$ is defined by (15).

Observe that $\lim _{j \rightarrow \infty} \omega_{j} \in(0,1]$, and, consequently, the sequence $\left(\left|\ln \omega_{j}\right|\right)_{j \in \mathbb{N}}$ is bounded. Since $b_{d} \rightarrow \infty, d \rightarrow \infty$, for every $\tau>0$ there exists $d_{\tau} \in \mathbb{N}$ such that $\left|\ln \omega_{j}\right| \leqslant \tau b_{d}$ holds for any $j \in \mathbb{N}$ and $d \geqslant d_{\tau}$. Therefore for every $\tau>0$ we have

$$
\sum_{\substack{j=1, \ldots, d \\\left|\ln \omega_{j}\right|>\tau b_{d}}} \omega_{j}=0, \quad \text { and } \quad \sum_{\substack{j=1, \ldots, d \\\left|\ln \omega_{j}\right| \leqslant \tau b_{d}}} \frac{\left|\ln \omega_{j}\right| \omega_{j}}{1-\omega_{j}}-\hat{a}_{d}=\sum_{j=1}^{d} \frac{\left|\ln \omega_{j}\right| \omega_{j}}{1-\omega_{j}}-\hat{a}_{d}=0
$$

for all sufficiently large $d$, i.e. we get (22) and (23). Next, we also have

$$
\frac{1}{b_{d}^{2}} \sum_{\substack{j=1, \ldots, d \\\left|\ln \omega_{j}\right| \leqslant \tau b_{d}}} \frac{\left|\ln \omega_{j}\right|^{2} \omega_{j}}{\left(1-\omega_{j}\right)^{2}}=\frac{1}{b_{d}^{2}} \sum_{j=1}^{d} \frac{\left|\ln \omega_{j}\right|^{2} \omega_{j}}{\left(1-\omega_{j}\right)^{2}}=\frac{1}{\rho^{2}} \cdot \frac{1}{d} \sum_{j=1}^{d} \frac{\left|\ln \omega_{j}\right|^{2} \omega_{j}}{\left(1-\omega_{j}\right)^{2}}
$$


for all sufficiently large $d$. The arithmetic mean in the right-hand side tends to $\lim _{j \rightarrow \infty} \frac{\left|\ln \omega_{j}\right|^{2} \omega_{j}}{\left(1-\omega_{j}\right)^{2}}=\rho^{2}$. Thus for every $\tau>0$ we get

$$
\lim _{d \rightarrow \infty} \frac{1}{b_{d}^{2}} \sum_{\substack{j=1, \ldots, d \\\left|\ln \omega_{j}\right| \leqslant \tau b_{d}}} \frac{\left|\ln \omega_{j}\right|^{2} \omega_{j}}{\left(1-\omega_{j}\right)^{2}}=1
$$

that implies (24).

We now proceed to the case $\sigma_{j} \rightarrow \infty$ as $j \rightarrow \infty$. Here the asymptotics of $n^{Y_{d}}(\varepsilon)$ depends on the velocity of $\sigma_{j}$. In order to evidently illustrate the application of the general results from the previous section and to avoid routine unwieldy calculations, we will assume that

$$
\sigma_{j}^{2} \sim \beta j^{\alpha}, \quad j \rightarrow \infty
$$

with some $\alpha>0$ and $\beta>0$.

The following assertion directly follows from Propositions 1 and 2.

Proposition 4 Let $\left(\sigma_{j}\right)_{j \in \mathbb{N}}$ satisfy (25) with some $\alpha>0$ and $\beta>0$. If $\alpha>1$ then $\sup _{d \in \mathbb{N}} n^{Y_{d}}(\varepsilon)<\infty$ for every $\varepsilon \in(0,1)$. If $\alpha \leqslant 1$ then $n^{Y_{d}}(\varepsilon) \rightarrow \infty$ as $d \rightarrow \infty$ for every $\varepsilon \in(0,1)$.

We now propose the asymptotics of $n^{Y_{d}}(\varepsilon)$ for the case $\alpha \in(0,1]$.

Proposition 5 Let $\left(\sigma_{j}\right)_{j \in \mathbb{N}}$ satisfy (25) with some $\alpha \in(0,1]$ and $\beta>0$. Then

$$
\forall \varepsilon \in(0,1) \quad \ln n^{Y_{d}}(\varepsilon)=a_{d}+\Phi^{-1}\left(1-\varepsilon^{2}\right) b_{d}+o\left(b_{d}\right), \quad d \rightarrow \infty,
$$

where $\Phi$ is the distribution function of the standard Gaussian law,

$$
a_{d}=\sum_{j=1}^{d}\left(\frac{\left|\ln \omega_{j}\right| \omega_{j}}{1-\omega_{j}}+\left|\ln \left(1-\omega_{j}\right)\right|\right), \quad \text { and } \quad b_{d}= \begin{cases}\frac{\alpha}{\sqrt{(1-\alpha) \beta}} d^{\frac{1-\alpha}{2}} \ln d, & \alpha \in(0,1), \quad d \in \mathbb{N} . \\ \frac{1}{\sqrt{3 \beta}}(\ln d)^{3 / 2}, & \alpha=1,\end{cases}
$$

Remark 3 The following asymptotic holds

$$
a_{d} \sim \begin{cases}\frac{\alpha}{(1-\alpha) \beta} d^{1-\alpha} \ln d+\frac{1}{(1-\alpha) \beta} d^{1-\alpha}, & \alpha \in(0,1), \\ \frac{1}{2 \beta}(\ln d)^{2}+\frac{1}{\beta} \ln d, & \alpha=1,\end{cases}
$$

Indeed, since $\sigma_{j} \rightarrow \infty$ as $j \rightarrow \infty$, we have $\omega_{j} \sim \sigma_{j}^{-2} \sim\left(\beta j^{\alpha}\right)^{-1}, j \rightarrow \infty$, due to (66) and (25). Then

$$
a_{d} \sim \sum_{j=1}^{d}\left(\frac{\alpha \ln j}{\beta j^{\alpha}}+\frac{1}{\beta j^{\alpha}}\right) \sim \frac{\alpha}{\beta} \int_{1}^{d} \frac{\ln x}{x^{\alpha}} \mathrm{d} x+\frac{1}{\beta} \int_{1}^{d} \frac{1}{x^{\alpha}} \mathrm{d} x, \quad d \rightarrow \infty .
$$

Using known asymptotic relations (see [1] and [14]), we obtain (26]).

More sharp asymptotics of $a_{d}\left(\right.$ up to $\left.o\left(b_{d}\right)\right)$ require additional assumptions on the asymptotics of the difference of $\sigma_{j}^{2}-\beta j^{\alpha}$ as $j \rightarrow \infty$. 
Proof of Proposition 5. As in Proposition 3, according to Theorem 2, it is sufficient to check conditions (22)-(24) with given $a_{d}, b_{d}$, and with $\hat{a}_{d}$ defined by (15), $d \in \mathbb{N}$.

Since $\omega_{j} \sim\left(\beta j^{\alpha}\right)^{-1}, j \rightarrow \infty$, it is not difficult to see that for every $\tau>0$

$$
\frac{1}{\tau b_{d}} \max _{j \in\{1, \ldots, d\}}\left|\ln \omega_{j}\right| \rightarrow 0, \quad d \rightarrow \infty
$$

Therefore for every $\tau>0$ we have

$$
\sum_{\substack{j=1, \ldots, d \\\left|\ln \omega_{j}\right|>\tau b_{d}}} \omega_{j}=0, \quad \text { and } \quad \sum_{\substack{j=1, \ldots, d \\\left|\ln \omega_{j}\right| \leqslant \tau b_{d}}} \frac{\left|\ln \omega_{j}\right| \omega_{j}}{1-\omega_{j}}-\hat{a}_{d}=\sum_{j=1}^{d} \frac{\left|\ln \omega_{j}\right| \omega_{j}}{1-\omega_{j}}-\hat{a}_{d}=0
$$

for all sufficiently large $d$, i.e. we get (22) and (23). Next, we also have

$$
\sum_{\substack{j=1, \ldots, d \\\left|\ln \omega_{j}\right| \leqslant \tau b_{d}}} \frac{\left|\ln \omega_{j}\right|^{2} \omega_{j}}{\left(1-\omega_{j}\right)^{2}}=\sum_{j=1}^{d} \frac{\left|\ln \omega_{j}\right|^{2} \omega_{j}}{\left(1-\omega_{j}\right)^{2}}
$$

for every $\tau>0$ and for all sufficiently large $d$. Using the asimptotics $\omega_{j} \sim\left(\beta j^{\alpha}\right)^{-1}, j \rightarrow \infty$, we get

$$
\sum_{j=1}^{d} \frac{\left|\ln \omega_{j}\right|^{2} \omega_{j}}{\left(1-\omega_{j}\right)^{2}}=\sum_{j=1}^{d} \frac{(\alpha \ln j+\ln \beta+o(1))^{2}}{\beta j^{\alpha}(1+o(1))} \sim \frac{\alpha^{2}}{\beta} \sum_{j=1}^{d} \frac{(\ln j)^{2}}{j^{\alpha}}, \quad d \rightarrow \infty .
$$

By known asymptotic relations (see [1] and [14]), we obtain

$$
\sum_{j=1}^{d} \frac{(\ln j)^{2}}{j^{\alpha}} \sim \int_{1}^{d} \frac{(\ln x)^{2}}{x^{\alpha}} \mathrm{d} x \sim \begin{cases}\frac{1}{1-\alpha} d^{1-\alpha}(\ln d)^{2}, & \alpha \in(0,1), \quad d \rightarrow \infty, \\ \frac{1}{3}(\ln d)^{3}, & \alpha=1,\end{cases}
$$

Hence for every $\tau>0$

$$
\sum_{\substack{j=1, \ldots, d \\
\left|\ln \omega_{j}\right| \leqslant \tau b_{d}}} \frac{\left|\ln \omega_{j}\right|^{2} \omega_{j}}{\left(1-\omega_{j}\right)^{2}} \sim\left\{\begin{array}{ll}
\frac{\alpha^{2}}{(1-\alpha) \beta} d^{1-\alpha}(\ln d)^{2}, & \alpha \in(0,1), \quad d \rightarrow \infty . \\
\frac{1}{3 \beta}(\ln d)^{3}, & \alpha=1,
\end{array} \quad d \rightarrow \infty\right.
$$

Thus

$$
\lim _{d \rightarrow \infty} \frac{1}{b_{d}^{2}} \sum_{\substack{j=1, \ldots, d \\\left|\ln \omega_{j}\right| \leqslant \tau b_{d}}} \frac{\left|\ln \omega_{j}\right|^{2} \omega_{j}}{\left(1-\omega_{j}\right)^{2}}=1, \quad \tau>0
$$

that implies (24).

There exist particular cases, where the $\varepsilon$-component of asymptotics (13) is a quantile of a nonGaussian self-decomposable distribution function. One such case is considered in the next proposition. There appear distribution functions $D_{\mu}, \mu>0$, of $\mu$-convolution powers of the Dickman law (see [9] and [1] ). It is known that $D_{\mu}$ has the spectral representation triplet $\left(\frac{\pi \mu}{4}, 0, L\right)$, where $L(x)=$ $\mu \ln x \mathbb{1}(x \in(0,1]), x \in \mathbb{R} \backslash\{0\}$. 
Proposition 6 Suppose that $\sigma_{j}^{2} \sim \beta j \ln j, j \rightarrow \infty$, with some $\beta>0$. Then

$$
\forall \varepsilon \in(0,1) \quad \ln n^{Y_{d}}(\varepsilon)=D_{1 / \beta}^{-1}\left(1-\varepsilon^{2}\right) \ln d+o(\ln d), \quad d \rightarrow \infty .
$$

Proof of Proposition 6. We use Theorem 2 with $a_{d}:=0, b_{d}:=\max \{\ln d, 1\}, d \in \mathbb{N}, G:=D_{1 / \beta}$, $c=\frac{\pi}{4 \beta}, v=0$, and $L(x)=\frac{1}{\beta} \ln x \mathbb{1}(x \in(0,1]), x \in \mathbb{R} \backslash\{0\}$. So it is sufficient to check the following ensemble of conditions:

$$
\begin{aligned}
& \lim _{d \rightarrow \infty} \sum_{\substack{j=1, \ldots, d \\
\left|\ln \omega_{j}\right|>\tau \ln d}} \omega_{j}=-\frac{1}{\beta} \ln \tau \mathbb{1}(\tau \in(0,1]), \quad \tau>0, \\
& \lim _{d \rightarrow \infty} \frac{1}{\ln d}\left(\sum_{\substack{j=1, \ldots, d \\
\left|\ln \omega_{j}\right| \leqslant \tau \ln d}} \frac{\left|\ln \omega_{j}\right| \omega_{j}}{1-\omega_{j}}+\sum_{j=1}^{d}\left|\ln \left(1-\omega_{j}\right)\right|\right)=\frac{\pi}{4 \beta}+\gamma_{\tau}, \quad \tau>0, \\
& \lim _{\tau \rightarrow 0} \frac{\lim }{d \rightarrow \infty} \frac{1}{(\ln d)^{2}} \sum_{\substack{j=1, \ldots, d \\
\left|\ln \omega_{j}\right| \leqslant \tau \ln d}} \frac{\left|\ln \omega_{j}\right|^{2} \omega_{j}}{\left(1-\omega_{j}\right)^{2}}=\lim _{\tau \rightarrow 0} \varlimsup_{d \rightarrow \infty} \frac{1}{(\ln d)^{2}} \sum_{\substack{j=1, \ldots, d \\
\left|\ln \omega_{j}\right| \leqslant \tau \ln d}} \frac{\left|\ln \omega_{j}\right|^{2} \omega_{j}}{\left(1-\omega_{j}\right)^{2}}=0 .
\end{aligned}
$$

Here

$$
\gamma_{\tau}=\frac{1}{\beta} \int_{0}^{\tau} \frac{x^{2} \mathbb{1}(x \in(0,1))}{1+x^{2}} \mathrm{~d} x-\frac{1}{\beta} \int_{\tau}^{+\infty} \frac{\mathbb{1}(x \in(0,1))}{1+x^{2}} \mathrm{~d} x, \quad \tau>0 .
$$

Due to the assumption, we have

$$
\omega_{j} \sim \sigma_{j}^{-2} \sim \frac{1}{\beta j \ln j}, \quad j \rightarrow \infty .
$$

We first check (27). It is easily seen that for $\tau>1$ we have $\left|\ln \omega_{j}\right| \leqslant \tau \ln d, j=1, \ldots, d$, for all sufficiently large $d$. Therefore (27) obviously holds in this case. For $\tau \in(0,1]$ we set

$$
j_{d, \tau}=\min \left\{j \in \mathbb{N}:\left|\ln \omega_{j}\right|>\tau \ln d\right\}, \quad d \in \mathbb{N} .
$$

Due to (30), it is not difficult to see that $j_{d, \tau} \leqslant d$ for all sufficiently large $d$ and $\ln j_{d, \tau} \sim \tau|\ln d|$, $d \rightarrow \infty$. Next, observe that

$$
\sum_{\substack{j=1, \ldots, d \\\left|\ln \omega_{j}\right|>\tau b_{d}}} \omega_{j}=\sum_{j=j_{d, \tau}}^{d} \omega_{j} \sim \sum_{j=j_{d, \tau}}^{d} \frac{1}{\beta j \ln j}, \quad d \rightarrow \infty
$$

Using the known asymptotics (see [14], 2.13, p. 21)

$$
\sum_{k=1}^{n} \frac{1}{k \ln k}=\ln \ln n+C+o(1), \quad n \rightarrow \infty
$$

with a constant $C$, we obtain

$$
\begin{aligned}
\sum_{j=j_{d, \tau}}^{d} \frac{1}{\beta j \ln j} & =\frac{1}{\beta}\left(\ln \ln d-\ln \ln j_{d, \tau}\right)+o(1) \\
& =\frac{1}{\beta}\left(\ln \ln d-\ln \ln d^{\tau}\right)+o(1) \\
& =-\frac{1}{\beta} \ln \tau+o(1), \quad d \rightarrow \infty
\end{aligned}
$$


Thus (27) holds.

We now check (28). Observe that for every $\tau>0$

$$
\begin{aligned}
\frac{\pi}{4 \beta}+\gamma_{\tau} & =\frac{\pi}{4 \beta}+\frac{1}{\beta} \int_{0}^{\min \{\tau, 1\}} \frac{x^{2}}{1+x^{2}} \mathrm{~d} x-\frac{1}{\beta} \int_{\min \{\tau, 1\}}^{1} \frac{1}{1+x^{2}} \mathrm{~d} x \\
& =\frac{\pi}{4 \beta}+\frac{1}{\beta} \int_{0}^{\min \{\tau, 1\}} \mathrm{d} x-\frac{1}{\beta} \int_{0}^{1} \frac{1}{1+x^{2}} \mathrm{~d} x \\
& =\frac{1}{\beta} \min \{\tau, 1\} .
\end{aligned}
$$

Next, due to (30) and (32), we have

$$
\sum_{j=1}^{d}\left|\ln \left(1-\omega_{j}\right)\right| \sim \sum_{j=1}^{d} \omega_{j} \sim \sum_{j=1}^{d} \frac{1}{\beta j \ln j} \sim \frac{1}{\beta} \ln \ln d=o(\ln d), \quad d \rightarrow \infty .
$$

Next, if $\tau>1$ then $\left|\ln \omega_{j}\right| \leqslant \tau \ln d, j=1, \ldots, d$, for all sufficiently large $d$. Therefore

$$
\sum_{\substack{j=1, \ldots, d \\\left|\ln \omega_{j}\right| \leqslant \tau b_{d}}} \frac{\left|\ln \omega_{j}\right| \omega_{j}}{1-\omega_{j}}=\sum_{j=1}^{d} \frac{\left|\ln \omega_{j}\right| \omega_{j}}{1-\omega_{j}} \sim \sum_{j=1}^{d} \frac{1}{\beta j} \sim \frac{1}{\beta} \ln d, \quad d \rightarrow \infty
$$

If $\tau \in(0,1]$ then, according to (31), we write

$$
\sum_{\substack{j=1, \ldots, d \\\left|\ln \omega_{j}\right| \leqslant \tau b_{d}}} \frac{\left|\ln \omega_{j}\right| \omega_{j}}{1-\omega_{j}}=\sum_{j=1}^{j_{d, \tau}-1} \frac{\left|\ln \omega_{j}\right| \omega_{j}}{1-\omega_{j}} \sim \sum_{j=1}^{j_{d, \tau}-1} \frac{1}{\beta j} \sim \frac{1}{\beta} \ln j_{d, \tau} \sim \frac{\tau}{\beta} \ln d, \quad d \rightarrow \infty .
$$

Therefore we have for every $\tau>0$

$$
\sum_{\substack{j=1, \ldots, d \\\left|\ln \omega_{j}\right| \leqslant \tau b_{d}}} \frac{\left|\ln \omega_{j}\right| \omega_{j}}{1-\omega_{j}} \sim \frac{1}{\beta} \min \{\tau, 1\} \ln d, \quad d \rightarrow \infty
$$

Thus (33), (34), and (35) together yield (28).

Next, we check the condition (29). Let us fix $\tau \in(0,1)$ and consider

$$
\sum_{\substack{j=1, \ldots, d \\\left|\ln \omega_{j}\right| \leqslant \tau b_{d}}} \frac{\left|\ln \omega_{j}\right|^{2} \omega_{j}}{\left(1-\omega_{j}\right)^{2}}=\sum_{j=1}^{j_{d, \tau}-1} \frac{\left|\ln \omega_{j}\right|^{2} \omega_{j}}{\left(1-\omega_{j}\right)^{2}} \sim \sum_{j=1}^{j_{d, \tau}-1} \frac{\ln j}{\beta j} \sim \frac{\left(\ln j_{d, \tau}\right)^{2}}{2 \beta} \sim \frac{\tau^{2}(\ln d)^{2}}{2 \beta}, \quad d \rightarrow \infty .
$$

Therefore

$$
\lim _{d \rightarrow \infty} \frac{1}{(\ln d)^{2}} \sum_{\substack{j=1, \ldots, d \\\left|\ln \omega_{j}\right| \leqslant \tau b_{d}}} \frac{\left|\ln \omega_{j}\right|^{2} \omega_{j}}{\left(1-\omega_{j}\right)^{2}}=\frac{\tau^{2}}{2 \beta} .
$$

From this we obtain (29). 


\section{Acknowledgments}

The work of A. A. Khartov was supported by RFBR-DFG grant 20-51-12004.

\section{References}

[1] N. H. Bingham, C. M. Goldie, J. L. Teugels, Regular Variation, Camb. Univ. Press, Cambridge, 1987.

[2] J. L. Brown, Mean Square truncation error in series expansions of random functions, J. Soc. Indust. Appl. Math., 8 (1960), 1, 28-32.

[3] M. D. Buhmann, Radial Basis Functions: Theory and Implementations, Cambridge University press, Cambridge, 2003.

[4] J. Chen, H. Wang, Average case tractability of multivariate approximation with Gaussian kernels, J. Approx. Theory, 239 (2019), 51-71.

[5] G. E. Fasshauer, F. J. Hickernell, H. Woźniakowski, Average case approximation: convergence and tractability of Gaussian kernels, in L. Plaskota, H. Woźniakowski (Eds.), Monte Carlo and Quasi-Monte Carlo 2010, Springer Verlag, 2012, 329-345.

[6] G. E. Fasshauer, F. J. Hickernell, H. Woźniakowski, On dimension-independent rates of convergence for function approximation with Gaussian kernels, SIAM J. Numer. Analysis, 50 (2012), $247-271$.

[7] B. V. Gnedenko, A. N. Kolmogorov, Limit Distributions for Sums of Independent Random Variables, Addison-Wesley, Cambridge, 1954.

[8] T. Hastie, R. Tibshirani, J. Friedman, Elements of Statistical Learning: Data Mining, Inference, and Prediction, second ed., in: Springer Series in Statistics, Springer, New York, 2009.

[9] D. Hensley, The convolution powers of the Dickman function, J. London Math. Soc., 33 (1986), no. $3,395-406$.

[10] A. Karol, A. Nazarov, Ya. Nikitin, Small ball probabilities for Gaussian random fields and tensor products of compact operators, Trans. Amer. Math. Soc., 360 (2008), no. 3, 1443-1474.

[11] A. A. Khartov, Asymptotic analysis of average case approximation complexity of Hilbert space valued random elements, J. Complexity, 31 (2015), 835-866.

[12] A. A. Khartov, A simplified criterion for quasi-polynomial tractability of approximation of random elements and its applications, J. Complexity, 34 (2016), 30-41.

[13] M. A. Lifshits, A. Papageorgiou, H. Woźniakowski, Average case tractability of non-homogeneous tensor product problems, J. Complexity, 28 (2012), 539-561.

[14] B. M. Makarov, M. G. Goluzina, A. A. Lodkin, A. N. Podkorytov, Selected Problems in Real Analysis, Transl. Math. Monog., vol. 107, AMS, Providence, Rhode Island, 1992. 
[15] E. Novak, H. Woźniakowski, Tractability of Multivariate Problems. Volume I: Linear Information, EMS Tracts Math. 6, EMS, Zürich, 2008.

[16] E. Novak, H. Woźniakowski, Tractability of Multivariate Problems. Volume III: Standard Information for Operators, in: EMS Tracts Math., vol. 18, EMS, Zürich, 2012.

[17] V. V. Petrov, Limit Theorems of Probability Theory: Sequences of Independent Random Variables, Oxford Stud. Prob., vol. 4, Clarendon Press, Oxford, 1995.

[18] C. E. Rasmussen, C. Williams, Gaussian Processes for Machine Learning, MIT Press, 2006.

[19] K. Ritter, Average-case Analysis of Numerical Problems, Lecture Notes in Math. No. 1733, Springer, Berlin, 2000.

[20] B. Schölkopf, A. J. Smola, Learning with Kernels: Support Vector Machines, Regularization, Optimization, and Beyond, MIT Press, Cambridge, Massachusetts, 2002.

[21] I. H. Sloan, H. Woźniakowski, Multivariate approximation for analytic functions with Gaussian kernels, J. Complexity, 45 (2018), 1-21.

[22] G. W. Wasilkowski, H. Woźniakowski, Average case optimal algorithms in Hilbert spaces, J. Approx. Theory, 47 (1986), 17-25.

[23] H. Wendland, Scattered Data Approximation, in: Cambridge Monographs on Applied and Computational Mathematics, Cambridge University Press, Cambridge, 2005.

[24] H. Zhu, C. K. I. Williams, R. J. Rohwer, M. Morciniec, Gaussian Regression and Optimal Finite Dimensional Linear Models, in C. M. Bishop (Edt.), Neural Networks and Machine Learning, Springer-Verlag, Berlin, 1998, 1-20. 